\title{
Toward an understanding of the influence of cultural background and domain experience on the effects of risk-pricing formats on risk perception.
}

\author{
Peter Fraser-Mackenzie, Ming-Chien Sung, Johnnie E.V. Johnson* \\ Centre for Risk Research, Southampton Management School, University of Southampton, \\ Southampton, SO17 1BJ, UK \\ *Corresponding author: Tel: +44 238059 2546, jej@soton.ac.uk
}

\begin{abstract}
Risk information framing can be a powerful tool for aiding the communication of risk and improving decision-making. However, little work has investigated the extent that these framing effects depend on the characteristics of the perceiver. In our study, we examine whether the effects of different risk-pricing formats on risky choices are the same for all individuals, no matter their domain experience or cultural background, or whether there are interactions between these factors. Survey 1 revealed that three risk-pricing formats of the same choice problem resulted in the same individuals making different risky choices (preference reversal); suggesting that risk-perception was distorted by the risk-pricing format manipulation. In Survey 2 , the effects of the risk-pricing formats were shown to differ by the participants' cultural background (Asian vs. European) and the extent of their domain experience. The fact that there were no differences between the cultural or domain experience groups in their overall tendency to select riskier (cf. safer) choices indicates that risk behavior differences between groups are often closely linked to perceptual, rather than simply attitudinal, cognitive processes. The results demonstrate the complex, interactive cognitive processes that are used to encode risk information, involving the framing of the information and the cultural background and previous experiences of the individual. We conclude that it is important to consider the characteristics of the individual (e.g., culture, domain experience, etc.) when manipulating riskinformation framing with the aim of improving their risk communication.
\end{abstract}

Key words: framing, culture, risk-information, domain-experience, risk-communication 


\section{Acknowledgement:}

We should like to thank Professor Michael Siegrist and the anonymous reviewers for their very helpful comments and suggestions on an earlier draft of this paper. 


\section{INTRODUCTION}

The presentation format of risk information (e.g., frequencies such as 1 in $5 \mathrm{vs.}$ probabilities such as 0.20 ) has been shown to play an important role in the perception of risk magnitude.(1-5) Accordingly, the choice between different types of risk-information formats "can be made according to the goal of the communication of risk" (Siegrist, 2006, p. 507).(6) In manipulating the manner in which information is presented, it is hoped that risk communication will be improved and the decision-maker will better percieve risks and make more informed decisions. However, it is unclear from the literature whether the effects of riskinformation formats are consistent for all types of individual. For example, individuals' risktaking behavior has been found to be influenced by differences in their nationality, ${ }^{(7,8)}$ gender, ( 9-11) genetic make-up(12) and even their criminality(13). Given these differences, can we be certain that framing effects are consistent across these different types of individual, and that the risk framing manipulations we make always influence the perception of risk in the manner intended? In other words, when considering the different types of risk-information formats, it may be necessary to consider both the "goal of the communication" as well as the characteristics of the perceiver.

A potential problem is that studies examining the effects of risk-information formats tend to recruit participants from only one type or group of individuals. Likewise, studies examining the differences in risk-taking between groups of individuals tend to employ only one risk-information format. This dissociation into group effects and framing effects is not a problem if the effects of these factors on risk-taking on different types of individual are truly independent. However, if this is not the case, then problems may arise. For example, a specific risk-information format may be tested on, and found to improve, university medical students' perception of medical risks. However, this may not work in a similar manner for the target layperson audience. Similarly, differences in risk-taking propensity between cultural groups (e.g., Chinese vs. Americans) observed using one risk-information format may disappear, or perhaps even reverse, if the information is presented differently. Such a finding might suggest that 
differences in risk-taking propensity between cultures is driven by perceptual, rather than attitudinal motivators. Such findings could have wide reaching impacts, for example, on international business negotiation strategies.(14)

Consider, via a simple example using hypothetical data, the possible impacts of two risk formats (Probabilities and Frequencies) on the risk taking propensity of different groups (Asians and Europeans). The data, summarized in Figure 1a, demonstrates that the riskinformation format and the group membership play a role in risk-taking propensity, but the effects are independent from each other. A simple explanation for this could be that the probabilities format increases risk-taking by affecting the perception of risk magnitude and Asian individuals tend to take more risks than Europeans. This latter effect may arise because of differences between the culture-defined groups in terms of risk attitude (i.e., Asians are more "risk-seeking" in nature ${ }^{1}$ ). However, if the data resulted in patterns such as those displayed in Figures $1 b-d$ then this would indicate that something more complex was occurring. In these cases, the differences between the culture-defined groups are observed to depend on the riskinformation format used and the effect of the risk-information on risk-taking depends on the group exposed to the frame.

Perhaps most significantly, if we observe patterns in risky choice behavior that are similar to those displayed in Figures 1b-d (cf. 1a), this would suggest that there exist differences between individuals from the two culture-defined groups in terms of their risk perception. Figure $1 \mathrm{~d}$ makes this particularly clear. Specifically, we cannot infer that Asians prefer to take more risks than Europeans (i.e., have more risk-seeking attitudes) because even though Asians take more risks under the frequency format, they take fewer risks under the probabilities format. Rather, in these circumstances, we could conclude that the perception of risk under different risk-information formats appears to depend on the cultural group - i.e., that a cultural difference in the encoding (i.e., perception) of risk information has occurred. Equally, we cannot

\footnotetext{
${ }^{1}$ It could also be because of systematic differences in risk perception (see Weber \& Hsee, 1998) - we cannot know for certain simply based on the evidence shown in Figure 1a.
} 
infer from Figure $1 \mathrm{~b}$ that the probabilities format always increases risk-taking (as observed for Asians) because risk taking by Europeans is reduced when the probabilities format is employed - again suggesting that cross-cultural differences in risk-perception can occur. Indeed, if the pattern displayed in Figure 1c was observed, we would have difficulty arguing that there are differences between Asians and Europeans in terms of their risk attitude because there are no such differences under the probabilities format.

[Figure 1 approx. here]

Our aim is to establish if risk-pricing formats act consistently on individuals' risk perception (i.e., independently from their group membership) or, as we suspect, whether the effects are dependent on group membership. In order to achieve this, we employed a repeatedmeasures design involving equivalent-mean risky choices in two surveys. We developed linearmixed models to assess the extent to which risk information is processed independently from, or is informed by, the cultural background and domain experiences of the individual.

The first survey presented the same seven risky choices (hypothetical two-horse races) three times to each participant using a different risk-pricing format each time (decimal returns, fractional odds and probabilities). Participants showed different risk-preferences associated with the same risky choices depending on the risk-pricing format presented. This suggests that the risk-pricing formats were biasing their risk perception. Further analysis suggested that this could have been because the different risk-pricing formats biased participants' perception of expected value, variance or skewness.

In the second survey, we assessed whether these effects were different depending on the characteristics of the individual (i.e. Asian vs. European and high vs. low gambling experience). We found that the effect of the risk-pricing formats on risk-choice behavior was indeed dependent on the cultural background and experience of the participants, confirming that risk perception is a complex process involving interactions between group effects (cultural background and gambling experience) and task feature effects (risk-pricing formats). Given that 
perception of risk depends on a combination of factors related to the individual as well as the task, we conclude that risk communications should carefully consider both the manner in which risk information is presented and the characteristics and experiences of the perceiver.

The paper proceeds as follows. In section 1.1 we review traditional and perceived riskattitude models and how they are used in the descriptive modeling of risky choices. In section 1.2 we introduce the specific risk-information formats and groups we examined and summarize the literature examining their influence on risk-taking behavior. We describe our two surveys (in sections 2 and 4, respectively) and how a linear mixed modeling methodology helps us to test our hypotheses: the first survey is designed to examine the degree and manner in which individuals' risk perception is biased by risk-pricing formats and the second survey is designed to assess whether or not these distortions are mediated by an individual's cultural background and domain experience. We examine the significance of the results of these two surveys in sections 3 and 5, respectively. We discuss all the results in section 6 and examine the implications of our work in the conclusion.

\subsection{The Traditional Risk-Return Model and the Perceived Risk-Attitude Model}

Markowitz (1959) was the first to propose the risk-return model, which defines an individual's willingness $W\left(X_{i}\right)$ to invest in a risky option $X_{i}$ as follows:(15)

$$
W\left(X_{i}\right)=f\left(E\left(X_{i}\right), R\left(X_{i}\right)\right)=E\left(X_{i}\right)-b R\left(X_{i}\right)
$$

where $E\left(X_{i}\right)$ and $R\left(X_{i}\right)$ are the expected value and risk (measured by the variance) of returns associated with option $X_{i}$. The parameter $b$ measures an individual's risk attitude, which determines how that risk is interpreted. $(7,14)$ In particular, when $b$ is $>/<0$ the individual is riskaverse/preferring, and the model predicts that the individual would have greater preference for low/high risk investments. Consequently, the willingness to invest is conceptualized as a tradeoff between the motives of greed (represented by the expected value) and fear (represented by the risk). 
However, there are a number of limitations associated with Markowitz's (1959) model, particularly with respect to the nature of $R\left(X_{i}\right)$ and the interpretation of $b$ in the risk-preference term, $b R\left(X_{i}\right)$. For example, it has been shown that variance may not accurately represent the way in which individuals truly perceive risk, and variance is only valid for quadratic utility functions. (16) Whilst these limitations are known, an adequate measure of perceived risk has not yet been developed.(17) One important reason for this is that individuals differ substantially in how they perceive risk magnitude,(18) just as they differ in the manner in which they perceive other information such as visual distance, (19) sound localization, (20) body sensations( ${ }^{(21)}$ and pain.(22)

In an attempt to overcome the limitations of variance for capturing risk perception, Weber and Milliman (1997) relaxed the assumption that perceived risk should be accounted for by a single index for all individuals in all situations. (23) They allowed perceived-risk to vary between individuals by replacing the variance with a personalized measure of perceived risk that was based on the individual's self-reported estimate of risk magnitude. In other words, Weber and Milliman (1997) did not treat perceived risk as a pre-defined index (e.g., $R\left(X_{i}\right)=$ $\operatorname{Var}\left(X_{i}\right)$ ) that was identical for each individual. Rather, the perceived-risk of individual $j$ could be an independent and personal function, $f$, of a risk index (e.g., $R_{j}\left(X_{i}\right)=f_{j}\left[\operatorname{Risk} \operatorname{index}\left(X_{i}\right)\right]$ ). In fact, cross-gender and cross-cultural differences in risk-preferences have been shown to be influenced by these self-reported risk magnitudes $\left(R_{j}\left(X_{i}\right)\right)$, rather than by differences in their risk-attitude coefficients $\left(b_{j}\right) \cdot(10,14)$

Figure 2 summarizes the elements of Markowitz's (1959) traditional risk-attitude model (TRAM) and Weber and Milliman's (1997) perceived risk-attitude model (PRAM). The TRAM assumes that risk perception equates to an index of risk (e.g., variance) for all individuals in all contexts. In addition, the TRAM includes a single risk-attitude coefficient to account for any differences in risk-preference between individuals. The PRAM, on the other hand, allows risk perception to vary between individuals, contexts, and cultures, independently from the riskattitude coefficient. This approach accommodates the results of studies which have shown that 
risk perception varies between individuals and can differ within individuals depending on the context of the choice. $(10,2310)$ Summarizing this work, Weber and Milliman (1997) concluded that, while "...[perceived-risk attitude] may be a stable personality trait... the effect of situational variables on choice may be the result of changes in risk perception" (p. 142).(23) Therefore, we argue it is important to account for differences within individuals in their perception of risk as a result of the potential contextual bias from situation $k$ (i.e., $R_{j k}\left(X_{i}\right)=f_{j k}\left[\operatorname{Risk}\right.$ index $\left.\left(X_{i}\right)\right]$, where $j$ denotes the individual and $X_{i}$ the option).

The key advantage of Weber and Milliman's (1997) PRAM is that it enables us to maintain the notion of human rationality, despite behavior contravening certain rational norms. For example, as will be explained in the next section, some superficial task-features can result in preference reversals in the judgment of risk information and these pose a challenge to a basic axiom of rationality; the notion of "invariance" or "extensionality".(24, 25) This axiom states that rational individuals should not change their choice preferences based on superficial changes to the task description. The notion of rationality of human intention can be retained if it is assumed that observed preference reversals result from biases in perception rather than from irrational changes in intention. In other words, the individual may have an invariant and rational attitude towards risk, indicative of their "stable personality trait" (Weber and Milliman, 1997, p.142). However, cognitive biases in the perception of the risks during the encoding of the risk information may result in the degree of risk being perceived as higher or lower (thus, explaining observed changes in preference).

As a result of the foregoing discussion and following the experimental work of Weber \& Milliman (1997), Weber \& Hsee (1998) and Weber, Blais, \& Betz (2002), we assume that for a given task, risk attitude, $b$, is invariant within individuals, but that it can vary between individuals ${ }^{2}$. This permits us to retain the fundamental assumption of human rationality by

\footnotetext{
${ }^{2}$ It is important to note that risk attitudes may not be stable across different types of task and domains. For example, Weber, Blais \& Betz (2002) reveal that an individual could be risk averse in some domains (e.g. financial decisions) but risk-seeking in others (e.g. health/safety, recreational, ethical, or social decisions).( ${ }^{10)}$ Nevertheless, given that we are only investigating a single task, we can assume an invariant risk attitude.
} 
assuming that preference reversals by the same individuals result from cognitive biases on riskperception.

[Figure 2 about here]

\subsection{Risk-Behavior Factors (RBFs)}

There are numerous risk-behavior factors (RBFs) that appear to influence risk-taking. Some factors relate more to the individual, such as their attitude to a certain type of risk, perhaps caused by their cultural upbringing or some personality trait or their gender. Other factors relate more to the situation in which the risk is being assessed, such as how that risk information is presented. In this study we examine three RBFs, the first is related to the situation in which the decision is taken, namely, risk-pricing formats (i.e., risk-information formats) and the other two are related to individual factors: cultural background and domain experience.

\subsubsection{Risk-Information Framing}

As discussed above, studies have demonstrated preference reversals due to superficial changes in the task description that distort individuals' risk perception. For example, Slovic, Monahan, \& MacGregor (2000) found that experienced forensic psychologists' and psychiatrists' evaluations of whether a patient should be released from hospital were affected by whether the risk they posed was presented in frequency or percentage formats. (3) To retain the assumption that these psychologists and psychiatrists acted with rational intentions, it is necessary to assume that the changes in their decisions were the result of distortions in their perception of risks rather than a result of a change in their intentions (i.e., attitudes).

The perception of choice information is not only affected by different modes of presentation but also by the magnitudes involved. For example, Loewenstein, Weber, Hsee, \& Welch (2001) observed that there is little difference in our evaluation of a 1 in 10,000 and a 1 in 10,000,000 chance of winning the lottery. (26) Wong \& Kwong (2005) also revealed that 
individuals place a higher value on an attribute that differentiates two alternatives when it is framed with small numbers (e.g., sound quality of .003\% vs. .01\%, in which the values indicate the percentage of noise in signal) rather than large numbers (e.g., sound quality of $99.997 \%$ vs. 99.99\%, in which the values indicate the percentage of 1 - (noise in signal)).(27) Similarly, prospect theory accounts for the fact that individuals tend to over/under weight low/high probabilities and to weight losses more heavily than gains(1). Consequerntly, framing alternatives in terms of losses (cf. gains) can result in different risky choice behavior. A key feature of prospect theory is the proposal that, when faced with a choice, individuals undertake an editing phase in which they use heuristics (e.g. rounding) to simplify the task. (28) Such heuristics will differ depending on the way the values are presented. Thus, the manner in which information is presented significantly impacts the way in which information is perceived, encoded, and then acted upon.

Weber and Milliman (1997) demonstrated a correlation between contextual factors that affect choices and factors that affect risk perception. (23) However, they did not show that accounting for individuals' biases in risk perception can improve predictions of their risky choice behavior. Consequently, we set out to test if this is the case; namely, to test Hypothesis 1:

H1: A model to predict risky choice behavior can be improved by including parameters to account for biases in risk perception.

We aim to induce these biases by presenting the same risk information to the same individuals but in different risk-pricing formats.

\subsubsection{Cultural Background and Domain Experience}

Studies have revealed that an individual's background can play an important role in their judgment and decision-making. For example, Asians have been shown to be more extreme (cf. Westerners) in their estimates of confidence when answering general knowledge questions, i.e., closer to certain or chance-guess ends of the confidence rating scale.(29,30) In addition, differences between those from different cultures have been found in the degree of 
disconfirmation in hypothesis testing( ${ }^{31)}$ and in susceptibility to cognitive biases related to financial investment. (32) Recent studies have also suggested that risk behavior can be predicted by genetic differences.(12) Consequently, it has been argued that risk perception ought to be modeled as a psychological variable which is subject to possible individual and cultural differences. $(7,14,33)$ This may be appropriate because the meaning and awareness of risk is related to social definitions and a cultural system of meaning. (34) In fact, studies have generally shown that Asians tend to take greater risks than Westerners. $(7,35)$ This is often explained by the "cushion hypothesis",(7) which proposes that individuals are more risk seeking if they have greater social support. It is argued that individuals from China can rely on greater social support due to the collectivistic culture, compared to the more individualistic culture found in the west (e.g. in US) and therefore feel more comfortable taking risks.

Studies have also suggested that cultural background may not only affect an individual's attitude to risk, but also the way in which they perceive risk. Indeed, using the PRAM approach described earlier, Weber and Hsee (1998) showed that greater risk-seeking behavior by Chinese (vs. American) decision makers resulted from differences in their perception of the investments' risk, $R\left(X_{i}\right)$, not from differences in their risk attitude parameters, $b .{ }^{(14)}$ Based on this literature, we might expect risks in our survey to be perceived to be lower by Asian participants and, therefore, for these individuals to make riskier choices.

However, whilst the literature discussed above suggests that there may be differences between individuals from different cultural groups in risk perception and/or attitude, it does not indicate whether or not the biasing effects of risk-pricing formats should be the same between different cultural groups. For example, are these group differences in risk taking increased or decreased or perhaps eliminated under certain risk-pricing formats?

In other research Bontempo, Bottom, \& Weber (1997) compared the risk judgments (related to monetary lotteries) of business students and security analysts from Hong Kong and Taiwan with those from the USA and the Netherlands. ${ }^{(8)}$ They found that the probability of loss and the magnitude of loss had a greater effect on perceived risk amongst the Western and Asian 
participants, respectively. However, there was no significant difference in risk perception between the different domain experience groups (students versus security analysts), suggesting that upbringing and environment shape the risk perception more than professional training or expertise. This null effect of domain experience on risk perception is surprising because expertise has been shown to affect how information is perceived and used to make non-risky choice decisions. For example, experts invariably develop a more detailed mental representation of alternatives and their associated attribute structure.( ${ }^{36)}$ Hence, experts tend to be better able to make use of choice attributes. $\left({ }^{37,38)}\right.$ Some studies have observed differences between experts and lay-people in their risk judgments and some have not.(39) In those that found effects, there was evidence of lower estimates of risk by experts (cf. lay people). (40-42) However, the extent that these differences are determined by variations in risk attitude or risk perception is unclear.

The above discussion suggests that culture and/or domain experience may have some influence on an individual's risk-taking behavior and we suspect that these factors may have an effect on risk perception and on the effect of risk-information formats on choice behavior. To explore this possibility we test Hypothesis 2:

\section{H2: Culture and domain experience mediate the distortive effect of risk-pricing}

formats.

If this is shown to be the case, it would add considerable weight to Weber and colleagues' argument that culture and experience are involved in the perceptual processes of encoding risk magnitude from choice information. More importantly, it would require a sophisticated modeling approach in which effect of different formats on risk perception may depend on the individual's background and experience.

\section{SURVEY 1}

An important aspect of the PRAM is that it provides a means for calculating the influence of various RBFs that can influence risky choice, including subjective expected value, $E\left(X_{i}\right)$, 
perceived risk (perhaps as a function of $R\left(X_{i}\right)$ ), and perceived-risk attitude, $b$. In order to test Hypothesis 1, we designed Survey 1 to examine the effect of risk-pricing formats on individuals' risky-choice preference reversal (i.e., within individual). Specifically, we consider the different risk-pricing formats commonly used to present risk in a gambling context and their distortive effect on risk perception: fractional odds (i.e., 3/1,5/4, etc.), which are often used in bookmaker markets, and decimal returns (e.g., $\$ 4, \$ 2.25$, etc.), which are usually found in pari-mutuel markets. Participants' risky choices, based on risk information presented using these pricing formats and a probability format, are compared. The probability format simply shows win likelihoods in a decimal form (e.g., .25, .60, etc.). This enables us to test if individuals perceive risks differently under these different formats and to observe the extent of any preference reversals. The pricing formats we compare are particularly significant because Gigerenzer \& Hoffrage $(1995,1999)$ found that people behave differently when presented with frequency rather than objectively equivalent probability data. ${ }^{(2,43)}$ In addition, Rottenstreich \& Kivetz (2006) proposed that modes which tend to elicit a non-probabilistic mindset (such as the decimal returns format) usually result in individuals making judgments that are less reliant on risk. (44) We would therefore expect that these formats may result in participants relying less on risk perception.

It should be noted that not all frequency formats improve decision-making and not all probability formats reduce performance. ${ }^{45)}$ It has been shown that normalized frequency formats, which do not easily convey the base rates, do not help elicit correct Bayesian reasoning and probability formats which mimic natural frequencies can elicit correct reasoning.(45) In our study, the fractional odds format is presented as a natural frequency between the two runners in each race (i.e., $5 / 4$ vs. $4 / 5$ for the longshot and favorite in one race). We consider this a natural frequency comparison because the odds show the expected number of wins for each horse out of the same baseline number of races (i.e. longshot expected to win 4 and favorite 5 out of 9 races). However, when comparing odds of, for example, the longshots in two different races (e.g. with odds of 5/4 and 2/1), the participant would need to compare different baseline 
numbers of races (i.e. baselines of 9 and 3 races respectively). This comparison would therefore be more difficult as it would not be made on the basis of natural frequencies. As such, the fractional odds format may help/not help comparison of runners within/between races.

The probabilities format, on the other hand, presents the chances of each horse on a scale between zero and one, simplifying between and within race comparisons. While, a simple probability (e.g., 0.8 vs. 0.2 for the favorite and longshot) may be encoded as a natural frequency by participants (e.g., chances perceived as 8 out of 10; and 2 out of 10) more complex probabilities (e.g., 0.9524 vs. 0.0476 ) may be rounded during the editing phase (see section 1.2.1) and be less accurately perceived. When presented with the returns format, the likelihoods must be calculated. This calculation is cognitively effortful and may also involve rounding during the editing phase. As such, we expect the relative winning chances of runners to be least well perceived under this format.

The current literature provides no clear indication of the cross-cultural differences that may occur in response to these different risk-information formats. However, in the literature review section (1.2.2) we discussed the "cushion hypothesis" and how this predicts Asian individuals to be more risk seeking in general. Yet the evidence from Weber and Hsee (1998) suggests that this may stem from differences in their perception of risk, rather than differences in their risk attitude. On this basis, we reason that if a format does not elicit a reliance on risk perception (i.e. returns format) we may not observe a difference between cultures in risk taking behavior. Whereas, formats that do elicit reliance on risk perception (i.e. probabilities format) may lead to cross-cultural differences such that Asian participants are more risk seeking than Europeans.

\subsection{Methodology}

\subsubsection{Participants and Procedure}

In total, 80 male and 62 female undergraduate student participants were recruited during a break in a class, with ages ranging from 19 to 41 years $(M=23.27, S D=4.28)$. They were allowed time to read a booklet containing an introduction, consent form, simple 
descriptions of the three risk-pricing formats, a general description of the two-horse races in which they would be asked to select either the favorite $(F:$ defined as the horse with the greater chance of winning, but with a corresponding lower payoff to a winning bet) or the longshot ( $L$ : defined as the horse least likely to win, but with a corresponding higher payoff to a winning bet). They were also given debriefing information, describing the nature of the survey. The participants were then presented with seven equivalent-mean risky-choices, namely, seven two-horse races in which the expected value of betting on the $F$ or the $L$ were identical. These races varied from one in which the winning chance of the $F$ was close to that of the $L$, to a race where the $L$ was very unlikely to win (cf. the $F$ ). Each participant was asked to select one horse in each of the seven races presented in each of the three different risk-pricing formats (i.e., to place a hypothetical unit stake bet on one of the horses in each race) and to record their decisions in the spaces provided; thus, each participant made 21 choices (see Table 1 for details). Blocks of races with the same risk-pricing formats were presented together but were counterbalanced between the participants. Thus, a participant may, for example, receive a block of seven 'probability-format' races, followed by seven 'odds-format' races, and finally seven 'returns-format' races. Before each group of races, a page described the subsequent risk-pricing format in detailed, yet simple, language so that the participants were clear about the meaning of the risk-pricing format (see appendix A1 for information given to participants). As a form of incentive, participants were instructed that each race would be simulated later that day, and the participant that won the most money as a result of their 'hypothetical' bets would be contacted via email for an unspecified reward (worth approximately \$30).

The order in which the three risk-pricing formats appeared was counterbalanced across the participants, leading to six different permutations. Furthermore, the order in which the races appeared within each risk-pricing format was also counterbalanced across all participants using a quasi-complete $7 \times 7$ Latin square. The final counterbalancing involved half the participants being presented with races where the $F$ was listed above the $L$ and the other half were presented with races where the $L$ was listed above the $F$. This counterbalancing produced 
84 permutations (3 (risk-pricing formats in various orders) by 7 (race order) by 2 (position of favorite/longshot)).

[Table 1 about here]

\subsubsection{Design}

As indicated above, all the seven races were presented using each of the three riskpricing formats. In the 'probabilities format', a horse's prospects were represented by its winning probability, $P$, and the participants were informed that should the horse win, then a $\$ 1$ bet would result in a payoff of $\$ 1 / P$; i.e., the original $\$ 1$ stake plus $\$(1 / P)-1$ profit (full details of the instructions given to participants are provided in Appendix A1). Participants were also informed that in the 'fractional odds format', which is the most common format employed by UK bookmakers, a horse's chance is represented as a ratio, $\mathrm{N}_{\text {Lose }} / N_{\text {Win }}$, namely, the number of occasions the horse is likely to lose $\left(\mathrm{N}_{\text {Lose }}\right)$, to the number of occasions it is likely to win $\left(N_{\text {Win }}\right)$, if the race were run $\mathrm{N}_{\text {Lose }}+\mathrm{N}_{\text {Win }}$ times. Consequently, the winning probability of a horse with odds $\mathrm{N}_{\text {Lose }} / N_{\text {Win }}$ would be $N_{\text {Win }} /\left(N_{\text {Win }}+\mathrm{N}_{\text {Lose }}\right)$. Finally, participants were informed that in the decimal returns format, which is widely used in pari-mutuel betting markets, a horse's prospects is represented in a single number, $Z$, such that a winning $\$ 1$ bet would result in a return of $\$ Z$ (i.e., the original stake plus $\$ Z-1$ profit). Consequently, the winning probability for this horse would be $1 / Z$.

The horseraces consisted of binary forced-choice, equivalent-mean, risky choices that required one of the two horses to be selected. The choice is between horses $C_{i}$ and $C^{\prime}{ }_{i}$, both choices having two possible outcomes: $C_{i}$ involving a payoff of $1 / P_{i}$ (with probability $P_{i}$ ) and a payoff of 0 (with probability $1-P_{i}$ ), written as follows:

$$
C_{i}=\left[P_{i}, 1 / P_{i} ; 1-P_{i}, 0\right]
$$

$C^{\prime}{ }_{i}$, on the other hand, has a payoff/probability structure as follows,

$$
C_{i}^{\prime}=\left[1-P_{i}, 1 /\left(1-P_{i}\right) ; P_{i}, 0\right]
$$


These horses (lotteries) have equivalent means but they differ in variance. It should be noted that unless $P_{i}=.5$ then there will always be a favorite and a longshot. In the event that $P_{i}$ is greater than .5 then $C_{i}$ and $C^{\prime}{ }_{i}$ are the safer (or favorite, $F$ ) and riskier (or longshot, L) options respectively, i.e., if, and only if, $C_{i}$ is the favorite, $C^{\prime}{ }_{i}$ is the longshot. Therefore, only $P_{i}$ is needed to differentiate the riskier and the safer choice in each race. Consequently, a meaningful index of the difference between the horses (lotteries) is the absolute difference between the probabilities associated with the gain outcomes from the two lotteries $C_{i}$ and $C^{\prime}{ }_{i} ;\left|P_{i}-\left(1-P_{i}\right)\right|$ (referred to as the lottery probability differential, or LPD). Note that the variance of the safer alternative in the equivalent-mean risk-choice is given as follows:

$$
\operatorname{Var}\left(C_{i}\right)=\sigma^{2}=\frac{1}{P_{i}}-1
$$

Note that $0<P_{i}<.5$ for the riskier choice and $1>P_{i}>.5$ for the safer choice. Thus, the variance for the riskier alternative in the equivalent-mean risky-choice is given as follows:

$$
\operatorname{Var}\left(C_{i}^{\prime}\right)=\sigma^{2}=\frac{1}{1-P_{i}}-1
$$

The variances of each of the choices in the seven races presented to participants are displayed in Table 1.

The difference between the longshot $C^{\prime}{ }_{i}$ and the favorite $C_{i}$ in terms of variance, or Lottery Variance Differential (LVD) is as follows, where a high $L V D_{i}$ indicates a choice in which the longshot has considerably more variance than the favorite;

$$
L V D_{i}=\operatorname{Var}\left(C_{i}^{\prime}\right)-\operatorname{Var}\left(C_{i}\right)
$$

It should be noted that an important characteristic of these choices is that they also differ in skewness and this difference can be captured by a Lottery Skew Differential (LSD). To calculate this, we denote $p_{h}$ as the probability of the gain outcome for a particular lottery (horse), $h$, and calculate the skewness function, $S\left(p_{h}\right)$, for lotteries $C_{i}$ and $C^{\prime}{ }_{i}$ (see appendix A2) as follows: 


$$
S\left(p_{h}\right)=\frac{\left(1-p_{h}\right)\left(1-2 p_{h}\right)}{p_{h}{ }^{2}}
$$

The skewness of each of the choices in the seven races presented to participants is displayed in Table 1. We define the difference in skewness between choices, as follows:

$$
L S D_{i}=S\left(1-P_{i}\right)-S\left(P_{i}\right)
$$

where $P_{i}$ is the probability of the favorite winning and therefore $1-P_{i}$ is the probability of the longshot winning.

A high value of $L S D_{i}$ indicates a choice in which the riskier choice is considerably more positively skewed than the safer choice. It has been shown that individuals often over-bet the longshot and under-bet the favorite at the racetrack (a phenomenon known as the favoritelongshot bias) and this is perhaps explained by an attraction to positive skewness.(47) Consequently, while individuals may experience aversion to increasing variance associated with the riskier choice in higher $L P D_{i}$ races, they may also be attracted to the greater positive skewness of the risker choice.

We define risk preference as simply the propensity to choose the riskier $(L)$ over the safer $(F)$ alternative at a given level of LPD. With this in mind, the risk preference for an individual may depend on the relative weighting of the negative impact of variance (assuming risk aversion) and positive impact of skewness (assuming positive-skewness preference) as $L P D_{i}$ increases. We cannot know beforehand what these weightings may be for each individual, yet it seems possible that those that tend to prefer the risker (safer) choice may place a greater weight on the skewness (variance) relative to the variance (skewness) attributes in that race. However, the nature of our equivalent-mean risky choices is such that there is a strong correlation ( $\mathrm{r}=0.970)$ between variance and skewness. This prevents us, due to issues related to multicollinearity, from examining the relative weighting that individuals apply to variance and skewness when making their choices.

Nevertheless, our main aim is not to establish this precise weighting. Rather, we are interested in determining if inconsistencies arise in risk preferences, however they form, within 
the same individual across different risk-pricing formats and what this might tell us about biases effects of these formats on risk perception. Therefore, it is important to note that, unlike the TRAM, we do not require our participants to perceive risk in terms of variance (see Figure 2). Rather, in our main analysis, we model each individual's risk-preferences as a function of LPD, where LPD simply serves as a reasonable reference of the risk level and it should be noted that as $L P D_{i}$ increases so does $L V D_{i}$ and $L S D_{i}$ (see Figure 3). Specifically, we use a linear mixed model to estimate the bias on risk perception from each risk-pricing format, while allowing for individual differences in risk preference across a range of races with different levels of $L P D_{i}$.

[Figure 3 approx here]

\subsection{Models}

We adapted Markowitz's (1959) risk-return model to a probabilistic binary choice model, estimating the probability of selecting the riskier choice over the safer choice $\operatorname{Pr}\left(X_{L}, X_{F}\right)$ as a tradeoff between alternatives ( $F$ and $L$ ), in terms of the differences in expected value, $E\left(X_{L}, X_{F}\right)$, and risk, $R\left(X_{L}, X_{F}\right)$ :

$$
\begin{gathered}
\operatorname{Pr}\left(X_{L}, X_{F}\right)=f\left\{W\left(X_{L}, X_{F}\right)\right\}=f\left\{\left[E\left(X_{L}, X_{F}\right)\right]-b\left[R\left(X_{L}, X_{F}\right)\right]\right\} \\
E\left(X_{L}, X_{F}\right)=E\left(X_{L}\right)-E\left(X_{F}\right) \\
R\left(X_{L}, X_{F}\right)=R\left(X_{L}\right) \quad R\left(X_{F}\right)
\end{gathered}
$$

According to this simple modification, choices may be predicted by a standard logistic function, $f$, of the willingness $(W)$ to choose $L$ over $F$.

\subsubsection{Model 1: Traditional-Risk Attitude Model}

For our seven lotteries $(i=1,2, \ldots, 7)$ we can present Eq.9 as a simple linear model as follows;

$$
\begin{gathered}
\operatorname{Pr}\left(X_{L}, X_{F}\right)=f\left\{\alpha+b R_{i}+\varepsilon_{i}\right\}, \\
R_{i}=\left[\mathrm{LPD}_{i}\right],
\end{gathered}
$$


where for choice $i$, the probability of selecting the riskier choice $\left(X_{L}\right)$ over the safer choice $\left(X_{F}\right)$ is estimated by as a logistic function, $f$, of the difference in the subjective expected value constant, $\alpha$, the risk attitude, $b$, and our risk difference index (represented by $L P D_{i}$ ) for race $R_{i}$. The subjective expected values can be represented by a constant because in the equivalentmean risky-choice designs all choices have the same expected value. However, it is clear that there may be individual differences in subjective expected value and in participants' risk preferences. As these are likely to be correlated within individuals over the repeated choices, we account for this in the models' error structures, $\varepsilon_{i}$, by employing a linear mixed model of the following form:

$$
\begin{aligned}
y_{j l}= & \beta_{1} x_{1 j l}+\beta_{2} x_{2 j l}+\cdots+\beta_{p} x_{p j l}+ \\
& \gamma_{j 1} z_{1 j l}+\gamma_{j 2} z_{2 j l}+\cdots+\gamma_{j q} z_{q j l}+\varepsilon_{j l}
\end{aligned}
$$

Or in a matrix form as,

$$
y=x \beta+z \gamma+\varepsilon
$$

where $y$ is the vector for the response variable for the lth observation by the $j$ th participant of a total of $m$ participants, $\beta_{1}, \ldots, \beta_{p}$ is the vector of fixed effects parameters, $x_{1 j l}$ is the vector for the first fixed effect regressor for observation $l$ by participant $j, \gamma_{j 1}, \ldots, \gamma_{j q}$, is the vector of random effect parameters for observation $l$ by participant $j$, and $z_{1 j l}$ is the vector for the first random effect regressor. In the TRAM, only one fixed factor is required beyond the vector for the intercept coefficient (the $L P D_{i}$ for choice $i$ ), as follows:

$$
\begin{aligned}
& x_{1,2, \ldots, p}=\left\{\left[\operatorname{LPD}_{i=1,2, \ldots, 7}\right]\right\} \\
& z_{1,2, \ldots, q}=\left\{\begin{array}{l}
{\left[\text { Participant }_{j=1,2, \ldots, m}\right],} \\
{\left[\text { Participant }_{j=1,2, \ldots, m} \times \operatorname{LPD}_{i=1,2, \ldots, 7}\right.}
\end{array}\right]
\end{aligned}
$$

This model is representative of the TRAM shown in Figure 2 because, when modeling risk-preference, it only allows for individual differences in subjective expected value $\left(a_{j}=\gamma_{j 1}\right.$ [Participant $]$ ] $)$ and risk attitude $\left(b_{j}=\gamma_{j 2}\left[\right.\right.$ Participant $\left.\left._{j} \times L P D_{i}\right]\right)$ and does not allow for the potential distortion of risk-preference under different risk-pricing formats, i.e., $R_{i}$ is simply equal to $L P D_{i}$. 


\subsubsection{Model 2: Perceived Risk-Attitude Model}

Hypothesis 1 implies that the TRAM may inadequately account for risky choices across the three $(k=1,2,3)$ different risk-pricing formats, as we expect the degree of perceived risk to vary by risk-pricing format, possibly leading to preference reversals. Consequently, we develop a PRAM, which retains the random factor variables from Model 1 (Eq. 15) but for the fixed factor variables we introduce an interaction term between the risk-pricing format (categorical factor) and the risk index (i.e., Risk-Pricing Format $\times L P D$ ), as follows:

$$
x_{1,2, \ldots, p}=\left\{\begin{array}{l}
{\left[\mathrm{LPD}_{i=1,2, \ldots, 7}\right],} \\
{[\text { Risk-Pricing Format }} \\
k=1,2,3 \\
{\left[\text { Risk-Pricing Format }_{k=1,2,3} \times \mathrm{LPD}_{i=1,2, \ldots, 7}\right]}
\end{array}\right\} .
$$

This allows us to model the bias in risk-perception caused by the risk-pricing format. We test Hypothesis 1 by determining, via a log likelihood ratio test (LLR), if the PRAM (Eq. 16), better captures individuals' risky choices than the simpler TRAM (Eq. 14).

\section{RESULTS}

\subsection{Descriptive Statistics}

The estimated choice behavior of each individual participant (thin grey lines) and the PRAM's estimation for the main effects (thick black lines) of each risk-pricing format are shown in Figure 4. We define risk-approach/-avoidance behavior as that where participants increase/decrease the proportion of risky choices as LPD increases. Consequently, the graphs in Figure 4 clearly show a significant degree of preference reversal arising from the different riskpricing formats. The odds format results in a considerable range of risk-approach and riskavoidance behavior amongst individuals but with an overall modest reduction in the proportion of riskier choices as LPD increases. The probability and returns formats elicit very different patterns of behavior. Most individuals reduce the number of riskier choices as LPD increases under the probability format and there is far less variation between individuals than under the 
odds format. However, most individuals tended to increase their proportion of risky choices as LPD increases under the decimal returns format.

\subsection{Inferential Statistics}

The results indicate that PRAM better accounted for the risky choices of the participants than TRAM $\left(\triangle \mathrm{AIC}-358.2, \chi^{2}\right.$ difference $\left.(4)=366.2, p<.001\right)$. The variable $L P D_{i}$ was a significant factor in $\operatorname{PRAM}(F(1,141)=9.62, p=.002)$, suggesting that the proportion of riskier (vs. safer) choices varied with $L P D_{i}$. Importantly, there was no main effect of the format $(F(2,2693)=0.69$, $p=.499)$ but rather a significant Risk-Pricing Format $\times L P D$ interaction $(F(2,2693)=34.33, p$ $<.001)$. As hypothesized, this interaction term indicates that, having controlled for individual differences in risk preference, risk was perceived by individuals as a function of $L P D$ and that this function differed depending on the framing of the risk-information (the risk-pricing format). Thus, we confirm Hypothesis 1 that the PRAM better accounts for risky choice behavior than the TRAM; the former model including and the latter model excluding parameters to account for biases in risk perception. These results suggest that to more accurately model participants' choices, it is important to account for biases in risk-perception within individuals resulting from risk-pricing format manipulations (i.e., to use the PRAM).

[Figure 4 about here]

These results are confirmed by the estimated random and fixed effects parameters for the PRAM (Table 2). The odds and probability pricing format interactions with LPD are both associated with negative risk-perception coefficients that are significant, whereas the returns format (shown by the reference level as LPD in Table 2) has a positive coefficient that is significant.

[Table 2 about here] 


\section{SURVEY 2}

Survey 2 was designed to test Hypothesis 2, namely, that culture and domain experience mediate the distortive effect of risk-pricing formats. Specifically, we explore whether culture and domain experience are only associated with differences in the overall proportion of risky choices (i.e., irrespective of the risk-pricing manipulation) or whether, in fact, these two RBFs play a role in mediating the biasing effects of risk-pricing format on risk perception within individuals. This is achieved by determining whether, as we expect, we are better able to model participants' risky choices if we allow culture and domain experience to mediate the effects of the risk-pricing formats on risk-perception rather than keeping the biasing effects of riskpricing formats separate from the culture/domain experience effects.

\subsection{Methodology}

\subsubsection{Participants and Procedure}

The participants from Survey 1 were also recruited for Survey 2. In total, 59 (42\%, males=28, females=31) reported themselves as being Chinese/Taiwanese nationality and 83 (58\%, males $=52$, females $=31$ ) reported themselves as being European. Participants were asked the total length of time they had spent in the UK. This was used to determine some quantifiable difference in the cultural experiences of the 'Asian' and 'European' groups. The median period the Asian and European groups reported they had been in the UK was 1 and 19 years, respectively.

We were concerned that gender differences may play a role in mediating the effect of pricing modes. However, tests revealed no significant difference between male and female participants in either risk preference or in the mediating effect of risk-pricing formats (see appendices A4 and A5). Furthermore, the difference in proportions of males and females in each group was not significant $\left(\chi^{2}(1)=2.65, p=.104\right)$. Consequently, we were confident that gender effects would not confound any observed cultural effects. 
A second potential confound could have arisen had there been significant differences in the education or mathematical ability of the Asian and European participants. Indeed, the different cultural groups were likely to have been educated in different educational systems. To limit this effect, all participants were recruited from the same MSc course in Risk Management, implying that they had all passed the rigorous University admissions procedures to confirm that they had exceeded a given level of educational attainment. Second, we asked all participants to undertake a simple arithmetic test based on probability theory. The results showed no significant difference in this test score $(t(115.86)=-0.89, p=.376)$ between the Asian (mean score $=3.16)$ and European (mean score $=3.45)$ participants.

Participants were also asked to report their gambling experience. In particular, they were asked their frequency of gambling on chance games (such as the national lottery, scratch cards, bingo, fruit machines, slot machines, roulette, or craps) and/or skill games (e.g., sports, horseracing, motor-racing, greyhound racing, poker, card games, or mahjong). The frequencies were reported on a 6-point scale ranging from 1, indicating 'never gamble', to 6 indicating 'daily gambling'.

\subsubsection{Design}

The participants were split into two groups based on their self-reported nationality: Asians (Chinese and Taiwanese) and Europeans (EU citizens). In addition, two groups were constructed on the basis of participants' self-reported domain experience, i.e., a gambling experience score. Participants with below and above mean gambling experience scores (based on all participants) were assigned to "lower" and "higher" domain experience groups, respectively. Thus, the participants were separated into four groups: Asian-Higher Experience (AHE), Asian-Lower Experience (ALE), European-Higher Experience (EHE), and EuropeanLower Experience (ELE). 


\subsection{Models}

The groupings based on cultural background and gambling experience were added as fixedfactors to the PRAM that was analyzed in Survey 1 (i.e. Model 2 introduced in section 2.2.2). In order to test Hypothesis 2, we compared the extent to which the risky choices of the participants could be adequately accounted for by two alternative models. The first, simpler model, isolated the cultural background and gambling experience RBFs from the risk-pricing format effects (Model 3: see below). The second model allowed the two RBFs to mediate the effects of the risk-pricing formats (Model 4: see below). Thus, Models 3 and 4 represent, respectively, the idea that the biasing effect of risk-pricing formats on choice behavior is independent of or dependent on, the two RBFs.

\subsubsection{Model 3: Independence Model}

It is possible that, in terms of factors affecting their risky choices, the only difference between individuals in the different culture or domain experience groups is their risk-attitudes; i.e., that the risk-pricing formats act in a similar fashion on individuals no matter what their cultural and domain experiences. This would simply mean that one culture or domain experience group tends to take more risks, but both are equally affected by the biasing effects of the risk-pricing formats on risk-perception. In order to capture this possibility, we developed Model 3, which is identical to Model 2 except that it includes an additional three way interaction: LPD $\times$ Culture $\times$ Experience. Thus, as in Model 2, LPD can interact with the Risk-Pricing Format (LPD ×RiskPricing Format) to capture the biasing effect of the formats on risk perception. However, this effect is modeled as an effect independent of the risk-preference functions for the four culture/gambling experience groups (modeled via the LPD $\times$ Culture $\times$ Experience interaction). Consequently, in Model 3 the effects of Risk-Pricing Formats are assumed to be independent of the group effects. As a result, the interactions in Model 3 are restricted to the following terms 3 :

\footnotetext{
${ }^{3}$ Only the highest level interactions are shown but all the lower order interactions and main effects are retained as indicated by “...”. Similarly, for eq. 20, all the lower order interactions and main effects are retained.
} 


$$
x_{1,2, \ldots, p}=\left\{\begin{array}{l}
{\left[\mathrm{LPD}_{i=1,2, \ldots, 7}\right],} \\
\ldots, \\
{\left[\mathrm{LPD}_{i=1,2, \ldots, 7} \times \text { Risk-Pricing Format }_{k=1,2,3}\right],} \\
{\left[\mathrm{LPD}_{i=1,2, \ldots, 7} \times \text { Culture }_{g=1,2} \times \text { Experience }_{m=1,2}\right]}
\end{array}\right\} .
$$

\subsubsection{Model 4 : Dependence Model}

If culture and domain experience mediate the biasing effect of the risk-pricing formats, we should observe a differential biasing effect of the pricing format among the four culture/domain experience groups. This can be captured by allowing further interactions to be included in the model as follows:

$$
x_{1,2, \ldots, p}=\left\{\begin{array}{l}
{\left[\mathrm{LPD}_{i=1,2, \ldots, 7}\right],} \\
\ldots, \\
{\left[\mathrm{LPD}_{i=1,2, \ldots, 7} \times \text { Risk-Pricing Format }_{k=1,2,3} \times \text { Culture }_{g=1,2}\right]} \\
{\left[\mathrm{LPD}_{i=1,2, \ldots, 7} \times \text { Risk-Pricing Format }_{k=1,2,3} \times \text { Experience }_{m=1,2}\right]}
\end{array}\right\}
$$

In this model, the LPD $\times$ Risk Pricing Format interaction (modeling the biasing effect of the risk-pricing formats) is allowed to interact with the two culture/gambling experience RBFs. This allows for a differential effect of the risk-pricing formats between the four groups (AHE, ALE, EHE, ELE). In this case, risk-pricing formats are assumed to be dependent on the cultural and domain experiences of the individual. Note that in both Models 3 and 4 we continue to control for individual differences in risk attitudes using the random variable regressors used in Models 1 and 2 (Eq. 15).

Consequently, Model 3 assumes that the risk-pricing formats have the same biasing effect on all groups, but that groups may vary in their risk-attitudes. Model 4 assumes that the risk-pricing formats may have a differential biasing effect depending on the culture and domain experience of the participants. Consequently, to test Hypothesis 2, we assessed, using loglikelihood ratio tests (LLR), whether Model 4 better accounted for participants' risky choices than Model 3. 


\section{RESULTS OF SURVEY 2}

There was a significant difference between the two cultural background groups in terms of the mean number of years that the group members had spent in the UK (Asian: $\mathrm{M}=1.69, \mathrm{SD}=1.44$ years; European: $\mathrm{M}=15.01, \mathrm{SD}=10.14$ years; $t(1,86.39)=11.83, p<.001)$. However, there was no significant difference between the participants in the two culture groups in terms of their average gambling experience: $\chi^{2}(1, N=142)=0.4029, p=0.526$. In addition, there was no significant difference in the proportion of riskier choices selected by Asian (M=42.7\%) and European $(M=46.1 \%)$ participants over the 21 races $(t(1,135.7)=0.942, p=0.347)$ or by those in the higher $(M=41.9 \%)$ and lower $(M=47.4 \%)$ gambling experience groups $(t(1,138.4)=1.523$, $p=0.13$ ). These results suggest that it is unlikely that there are any differences between the individuals in these groups in terms of their risk attitudes, i.e., in their general propensity to choose the riskier alternative.

The degree to which models 3 and 4 accounted for the risky choices of the participants was compared using LLR tests. The results, displayed in Table 3, reveal that Model 3 did not better account for the risky choices of participants than the PRAM (Model 2). This suggests that the culture and domain experience of the participants did not impact on their risky choice behavior independently of the risk-pricing formats. Model 4, on the other hand, was significantly better at accounting for the risky choices of participants than Model 3. This result supports Hypothesis 2, indicating that culture and/or domain experience impact on the effect of the risk-pricing formats on risk-perception.

[Table 3 approx here]

ANOVA results for Model 4 are shown in Table 4. An important finding was that the LPD $\times$ Culture and the LPD $\times$ Experience interactions were not significant. This shows that there is no significant difference between the cultural or domain experience groups in terms of their risk preferences once we control for effects of risk-pricing formats on risk perception. In other words, there is no significant difference in the behavioral responses (changes in the degree of risk-taking) to the underlying LPD of the choices between Asians and Europeans or between 
individuals with high and low gambling experience. Rather, the results suggest that the differences in risky choice behavior between individuals in the Asian and Europeans groups and between the high and low gambling experience groups appear to be associated with differences in their responses to the risk-pricing formats.

The results displayed in Table 4 also show that the LPD $\times$ Risk Pricing Format $\times$ Culture interaction is significant. This suggests that the effect of the risk-pricing formats on the change in the proportion of risky choices (as LPD increases), is different for each culture group. In addition, the Risk Pricing Format $\times$ Experience interaction is significant, whereas the LPD $\times$ Risk Pricing Format $\times$ Experience interaction is not significant. Accordingly, we simplify Model 4 to a best fitting version (Model 5) by removing the latter interaction:

$$
x_{1,2, \ldots, p}=\left\{\begin{array}{l}
{\left[\mathrm{LPD}_{i=1,2, \ldots, 7}\right],} \\
\ldots, \\
{\left[\text { Risk-Pricing Format }_{k=1,2,3} \times \text { Experience }_{m=1,2}\right]} \\
{\left[\mathrm{LPD}_{i=1,2, \ldots, 7} \times \text { Risk-Pricing Format }_{k=1,2,3} \times \text { Culture }_{g=1,2}\right]}
\end{array}\right\}
$$

This model is significantly better than Model 2 in explaining the risky choices of participants ( $\triangle \mathrm{AIC}-16.9, \chi^{2}$ difference $(4)=22.87, p<.001$ ), and it is the most parsimonious model of how culture and domain experience mediate the effect of the risk-pricing formats. The parameters of Model 5 are shown in Table 5 and the main effects are shown in Figure 5(a-c). Under the odds pricing format the 'high experience' participants tended to take more risks overall and Asian participants showed a greater risk-averse response to an increase in the LPD. A different pattern emerged for the probabilities pricing format. Here Europeans were more risk-preferring when the LPD was low (intercept at 0.68 (high experience) and 0.74 (low experience) compared to 0.36 and 0.42 , respectively, for Asians). However, both Asians and Europeans and individuals with high and low experience converged to almost zero risk-taking when the LPD approached one. Under the returns pricing format the pattern changes again. 
Here the participants with greater experience tended to take more risks and both Asian and European participants increased their degree of risk-taking as LPD increased.

[Tables 4 and 5 and Figure 5 about here]

In summary, the results clearly show that the differences between the Asian and European groups and between the high and low experience groups are not in their overall risktaking or in the changes in their degree of risk-taking in response to increased LPD. Rather the differences between the groups are captured by differences in the way that they respond to the three risk-pricing formats. In other words, at least in our study, culture and domain experience do not appear to be associated with differences in risk-attitudes or any general propensity to take more or less risk. Rather, the differences appear to be related to how the individuals in the groups perceive, encode and then act upon risk information framed by the risk-pricing format.

The results were consistent with our expectations that no differences in choice behavior would be observed with the returns pricing format, possibly because this format does not elicit a probabilistic mindset. However, contrary to our expectations based on the extant literature, we did not observe Asian participants selecting the riskier choice more frequently than European participants for pricing formats in which we expected a probabilistic mindset to be elicited (i.e. probabilities format). In fact, the significant negative Format(Probabilities) $\times$ Culture(Asian) coefficient in Table 5 indicates a significantly lower proportion of longshot choices selected by Asian participants (compared to Europeans) under the probabilities format.

\section{DISCUSSION}

Our results showed that risk approach/avoidance was strongly dependent on the riskpricing formats used to present the choice information. As such, risk preferences appear to arise from complex co-dependent processes involving the individual's risk attitude in a given task and also the encoding of risk, which appears to be highly dependent on the way the information is presented. We did not reveal any difference between Asians and Europeans or between the higher and lower domain-experience groups in terms of their overall tendency to select riskier 
choices (i.e., on average, across all of the risk-pricing format). Instead, we found that the distortive effect of the risk-pricing formats on choice behavior appeared to depend on the cultural background and domain experience of the participants. In particular, all groups were similarly risk-avoidant when presented with the probability pricing format. However, the fractional odds format resulted in comparatively greater risk-preference in Asians (cf. Europeans). In addition, the fractional odds and decimal returns formats resulted in greater risk preference amongst participants with greater gambling-experience.

Based on extant literature, we expected greater risk-taking by Asian participants when presented with pricing formats in which a probabilistic mindset is elicited (i.e. probabilities format). In fact, our results showed the opposite effect. One explanation for this finding is that our Asian participants were making these decisions while in residence in another country where they may feel more isolated from their "social cushioning". Without feeling the support from such cushioning, these individuals may have a heightened risk aversion. Indeed, Weber, Hsee and Sokolowska (1998), following from the concerns expressed by McDaniels and Gregory's (1991), discuss the importance of considering the impact of situational factors (i.e. current political and economic environments) as well as cultural norms in understanding crosscultural differences in risk taking. ${ }^{(35,46)}$ This highlights the main argument of this paper, that it is important to consider all the RBFs that might be involved in risky decision-making and how they might interact together to influence choice behavior.

The results demonstrate the important effects of risk-pricing formats employed in surveys and question the extent to which we can draw conclusions, particularly regarding an individual's true risk attitude, on the basis of studies using only one risk-pricing format. Our results suggest this is particularly important when employing experimental results to infer broader cultural and environmental differences in risk-taking. For example, it has been found that risk-preferences vary depending on culture but not domain experience.(8) Our results demonstrate that differences between groups in risk-taking can be heavily reliant on the nature of the task, as even superficial changes in the presentation of risk information can result in 
exposing or removing group differences in risk-taking. This leads us to question the extent that other demographic differences in risk-taking observed in the literature are truly attitudinal or simply the result of differences in risk perception and whether such differences may be removed or highlighted by assessing them under different task frames.

Of course, there are some very important caveats related to our study. Our participants were students, many of whom had traveled to the UK to study, whereas the participants in Bontempo et al.s' (1997) study were students who were living in their own respective coutries. In addition, our domain experience groups were based on self-reported measures of gambling experience, whereas the Bontempo et al. (1997) study used security analysts for the higherexperience group and university students for the lower-experience group. Consequently, our results cannot be compared directly and we are even cautious about infering too far beyond the boundaries of our study. The potential for sampling bias means that we cannot conclude that the effects shown are representative of all Asians and Europeans or all experienced and novice gamblers. Rather, our conclusion is more fundamental; namely, that we should be cautious in concluding that broad group differences/similarities exist without controling for the taskfeatures used in the study (e.g., risk information frames). Whether or not the differences we observe are truly the result of cultural experiences and gambling experience or whether they arose from other differences between the groups we cannot be certain. However, we can conclude that the risk-taking of different groups of individuals can be differentially affected by superfical manipulations of task framing. Given our repeated-measures design, we can also conclude that such effects are closely linked to how individuals encode and percieve the risk information. This conclusion is reached because the effects of the risk-pricing formats were observed to result in preference reversals by the same individuals even when differences between individuals in risk attitude were controlled in the model. Therefore, in summary, our results support the ideas of Weber and colleagues that risk perception is an important factor in understanding the many possible differences between groups of individuals in risk-taking behavior. 
Our use of linear mixed models allowed us to produce and plot estimations for each individual's subjective expected value and risk-preference (see Figure 4). By plotting the individual effects, as well as the main effects, we were able to show the impact of the riskpricing format manipulations on each individual participant in the study. As a result, we were able to show that while the fixed effects are important, the random effects between individuals are considerable. This suggests that an exploration of further RBFs is needed to help unravel the causes behind the variation in subjective expected values and risk-preferences between individuals.

A further benefit of our approach is that previous studies have modeled perceived-risk attitudes by asking participants to provide a self-reported measure of risk-magnitude (e.g., Weber \& Hsee, 2000). This methodology relies on the validity of introspection and the idea that the true value of perceived-risk is accessible to the participant's conscious mind. However, this notion is not in line with some of the dual-process theories of risk, which suggest that risk processing often involves automatic and subconscious mental processes.(26) Our approach of using a repeated-measures design and linear mixed modeling does not suffer from this limitation. Consequently, we believe that this provides a useful paradigm for undertaking perceived-risk attitude research.

While not the main focus of this study, it would have been interesting to explore the extent to which our results could be explained by how saliently the skewness and variance features of each choice were perceived under the different risk-pricing formats. However, the nature of the equivalent-mean risky choices we employ is such that there is a strong correlation between variance and skewness. This prevented us from separating the effects of these two moments. Future work examining to what extent individuals, when faced with different riskinformation formats, make choices which are affected by their personal characteristics (e.g., culture, domain experience, etc.), might employ lotteries with payoffs and probabilities designed such that skewness and variance are not highly correlated.(48) This would enable an 
assessment to be made of the extent to which these different moments were perceived under the different risk-formats.

We are particularly interested in the possibility of extending the perceived-risk attitude approach to capture different perceived-risk functions of a given risk index. We expect that this may involve more complex modeling than our simple linear model. Weber (2010), for example, suggests that combining the risk-return approach with concepts from cognitive psychology or psychophysics might be a useful approach to this problem. ${ }^{49)}$ Another alternative might be to consider mixture models and to determine whether these different perceptions of risk might be representative of a switching between different decision models for different tasks or where different models apply to different types of individual (see, Harrison \& Rutström, 2009).(50)

A potential limitation of our work is that participants may not have understood some of the pricing formats as well as others and this may have influenced their choices. To reduce this possibility we made every attempt to provide participants with clear explanations, with practical examples of how chances and payoffs could be calculated in each case. We also offered participants the opportunity to seek clarification of details of any of the pricing formats (see appendix A1 for details). Interestingly, at real world race tracks, sometimes fractional odds are employed (e.g. by UK bookmakers) and sometimes the returns format is used (in pari-mutuel markets) without explicit explanations being given to bettors. The fact that we find differences in choices based on these different pricing formats (whether derived, as we believe, from perceptual biases, or through lack of understanding on the part of bettors) presents some interesting implications for understanding discrepancies in the degree of favorite-longshot bias which have been observed in bookmaker and pari-mutuel markets. This topic is beyond the scope of this study but offers the prospect of valuable future research.

Our study highlights important differences in framing effects between individuals from different cultural backgrounds and between those with different levels of domain experience. However, our study did not aim to identify the cognitive mechanisms which might be behind these effects. Future research aimed at establishing these mechanisms might consider sampling 
from individuals residing in the countries being analyzed( ${ }^{(8)}$ and our findings suggest that a large number of RBF's should be included to account for as many potential confounds as possible.

The topic of incentives in economic experiments has attracted considerable attention and continues to be a debated topic.(51) It could be argued that the "unspecified", "winner-takes-

all" incentive scheme employed in our study may have promoted a maximal risk-taking strategy. However, our results suggest that the participants did not respond in this manner to the mild incentive as only 2 out of the 142 participants (1.4\%) surveyed, employed the maximal risk strategy (i.e. choosing the longshot alternative in each race). However, to further the understanding of the effects of the risk-pricing manipulations, a carefully incentivized, experimental approach such as a random-lottery incentive system ${ }^{(51-54)}$ would be desirable.

Despite this lack of clear incentivization, we do know that participants in our study were not simply choosing randomly. If this were the case, it would be very unlikely that we would observe any systematic effects of the pricing formats. It is possible that different incentive structures may have impacted on the effect sizes we observe, just as time pressure could be expected to increase the effect sizes. Indeed, we expect that the impact of framing on risky choices may depend on a complex pattern of interactions between numerous factors (e.g., the individual's risk preferences, domain experience, cultural norms, the presence of time pressure, incentives/payoff structures, stress etc.) and more research is needed to establish these interactions.

\section{CONCLUSION}

In conclusion, our results indicate that RBFs can play a complex, interactive role in risky decision-making. This is important because some risk-pricing formats resulted in no differences in risky choice behavior between groups of individuals (based on cultural background and experience) whereas other pricing formats resulted in large differences. Importantly, we found that there was no difference between groups in terms of their attitudes to risk, i.e., as defined by their overall propensity to be risk-seeking/avoidant, once the effect of the risk-pricing formats 
had been controlled in the model. Rather, the differences between the groups appeared to stem from differences in how they encoded risk-information presented in a particular manner. Awareness of this is important because, as Weber \& Hsee (1998, p.1216) point out, "[d]rawing the wrong inference from observed cultural differences in preference among a set of risky options (e.g., assuming that a negotiation partner from a different culture is risk-seeking when, in fact, she simply perceives the associated risks to be smaller) can lead to wrong predictions of her preferences in subsequent situations with different option sets". (14)

Ultimately, therefore, our study indicates that one should be cautious in assuming attitudinal differences between individuals when perceptual differences may better explain the data. In such cases, interventions aimed at tackling an individual's risk attitude may not be effective or may even be harmful. Rather, interventions aimed at tackling risk perception may be more fruitful. In particular, our study shows that those who seek to communicate information concerning risk must consider the target user group and its characteristics carefully. Risk communication that is effective for one group of individuals may not work in a similar fashion for others and, at worse, could even be detrimental to their decision-making. For example, the design of risk communication to aid forecasting, in the form of risk-information dashboards, may need to be different for executives and factory workers. $(55,56)$ Similarly, disease or treatment-risk information for doctors may need to differ from that designed to inform lay-people( ${ }^{57)}$ and odds pricing at the race-track may need to be presented in different formats to account for groups with different levels of experience or from different cultural backgrounds.(58)

Finally, we believe that the risk-return conceptualization of risk preference combined with equivalent-mean risky choices provides a highly controlled environment for experimental work exploring risk-taking. We also believe that an approach which combines this with repeated-measures designs and linear mixed models, as employed here, offers the prospect of providing us with a more vivid picture of both the main effects and the finer-grained, individual level effects of RBFs on risk-taking behavior. 


\section{REFERENCES}

1 Tversky A, Kahneman D. The framing of decisions and the psychology of choice. Science, 1981;211(4481):453-458.

2 Gigerenzer G, Hoffrage U. How to improve Bayesian reasoning without instruction: Frequency formats. Psychological Review, 1995;102(4):684-704.

3 Slovic P, Monahan J, MacGregor DG. Violence risk assessment and risk communication: The effects of using actual cases, providing instruction, and employing probability versus frequency formats. Law and Human Behavior, 2000;24(3):271-296.

4 Slovic P, Finucane ML, Peters E, MacGregor DG. Risk as analysis and risk as feelings: Some thoughts about affect, reason, risk, and rationality. Risk Analysis, 2004;24(2):311-322.

5 Slovic P. Informing and educating the public about risk. Risk analysis, 1986;6(4):403-15.

6 Siegrist M. Communicating low risk magnitudes: incidence rates expressed as frequency versus rates expressed as probability. Risk Analysis, 1997;17(4):507-10.

7 Weber E, Hsee C. Culture and individual judgment and decision making. Applied Psychology, 2000;49(1):32-61.

8 Bontempo RN, Bottom WP, Weber EU. Cross-Cultural differences in risk perception: A Model-Based approach. Risk Analysis, 1997;17(4):479-488.

9 Byrnes JP, Miller DC, Schafer WD. Gender differences in risk taking: A meta-analysis. Psychological Bulletin, 1999;125(3):367.

10 Weber EU, Blais AR, Betz NE. A domain-specific risk-attitude scale: Measuring risk perceptions and risk behaviors. Journal of Behavioral Decision Making, 2002;15(4):263290.

11 Fellner G, Maciejovsky B. Risk attitude and market behavior: Evidence from experimental asset markets. Journal of Economic Psychology, 2007;28(3):338-350.

12 Kuhnen CM, Chiao JY. Genetic determinants of financial risk taking. PLoS One, 2009;4(2):e4362.

13 Pachur T, Hanoch Y, Gummerum M. Prospects behind bars: Analyzing decisions under risk in a prison population. Psychonomic Bulletin \& Review, 2010;17(5):630-636.

14 Weber EU, Hsee C. Cross-cultural differences in risk perception, but cross-cultural similarities in attitudes towards perceived risk. Management Science, 1998;44(9):12051217.

15 Markowitz H. Portfolio selection. The Journal of Finance, 1952;7(1):77-91.

16 Weber E, Shafir S, Blais AR. Predicting risk-sensitivity in humans and lower animals: Risk as variance or coefficient of variation. Psychological Review, 2004;111:430-445. 
17 Luce RD, Weber EU. An axiomatic theory of conjoint, expected risk. Journal of Mathematical Psychology, 1986; 30(2):188-205.

18 Barnett J, Breakwell GM. Risk perception and experience: hazard personality profiles and individual differences. Risk Analysis, 2001;21(1):171-178.

19 Jackson RE. Individual differences in distance perception. Proceedings of the Royal Society B: Biological Sciences, 2009;276(1662):1665-1669.

20 Wenzel E, Wightman F, Kistler D, Foster S. Acoustic origins of individual differences in sound localization behavior. The Journal of the Acoustical Society of America, 1988;84:S79.

21 Steptoe A, Vögele C. Individual differences in the perception of bodily sensations: the role of trait anxiety and coping style. Behaviour Research and Therapy, 1992; 30(6):597-607.

22 Diatchenko L, Slade GD, Nackley AG, Bhalang K, Sigurdsson A, Belfer I, et al. Genetic basis for individual variations in pain perception and the development of a chronic pain condition. Human molecular genetics, 2005;14(1):135-43.

23 Weber EU, Milliman RA. Perceived risk attitudes: Relating risk perception to risky choice. Management Science, 1997;43(2):123-144.

24 Tversky A, Kahneman D. Rational choice and the framing of decisions. Journal of Business, 1986:S251-S78.

25 Arrow KJ. Risk perception in psychology and economics. Economic Inquiry, 1982;20(1): 19.

26 Loewenstein GF, Weber EU, Hsee CK, Welch N. Risk as feelings. Psychological Bulletin, 2001;127(2):267.

27 Wong KF, Kwong JY. Comparing two tiny giants or two huge dwarfs? Preference reversals owing to number size framing. Organizational Behavior and Human Decision Processes, 2005; 98(1):54-65.

28 Levy J. An introduction to prospect theory. Political Psychology, 1992;13(2):171-186.

29 Wright GN, Phillips LD. Cultural variation in probabilistic thinking: Alternative ways of dealing with uncertainty. International Journal of Psychology, 1980;15(1-4):239-257.

30 Phillips LD, Wright C. Cultural differences in viewing uncertainty and assessing probabilities: Springer; 1977.

31 Yates JF, Lee JW, Shinotsuka H. Beliefs about overconfidence, including its cross-national variation. Organizational Behavior and Human Decision Processes, 1996;65(2):138-147.

32 Kim KA, Nofsinger JR. Behavioral finance in Asia. Pacific-Basin Finance Journal, 2008;16(1):1-7.

33 Dake K. Orienting Dispositions in the Perception of Risk An Analysis of Contemporary Worldviews and Cultural Biases. Journal of Cross-Cultural Psychology, 1991;22(1):61-82. 
34 Abt V, McGurrin MC. Commercial gambling and values in American society: The social construction of risk. Journal of Gambling Studies, 1992;8(4):413-420.

35 Weber EU, Hsee CK, Sokolowska J. What folklore tells us about risk and risk taking: Crosscultural comparisons of American, German, and Chinese proverbs. Organizational Behavior and Human Decision Processes, 1998;75(2):170-186.

36 Hutchinson JW, Raman K, Mantrala MK. Finding choice alternatives in memory: Probability models of brand name recall. Journal of Marketing Research, 1994:441-461.

37 Hutchinson WJ, Eisenstein EM, Alba JW. Consumer learning and expertise. Handbook of Consumer Psychology, 2008:103-31.

38 Spence MT, Brucks M. The moderating effects of problem characteristics on experts' and novices' judgments. Journal of Marketing Research, 1997:233-247.

39 Rowe G, Wright G. Expert opinions in forecasting: The role of the Delphi technique. International Series in Operations Research and Management Science, 2001:125-144.

40 Flynn J, Slovic P, Mertz CK. Decidedly different: Expert and public views of risks from a radioactive waste repository. Risk Analysis, 1993;13(6):643-648.

41 Barke RP, Jenkins-Smith H, Slovic P. Risk perceptions of men and women scientists. Social Science Quarterly, 1997;78(1):167-176.

42 Slovic P, Malmfors T, Mertz CK, Neil N, Purchase IF. Evaluating chemical risks: results of a survey of the British Toxicology Society. Human \& Experimental Toxicology, 1997;16(6):289-304.

43 Gigerenzer G, Hoffrage U. Overcoming difficulties in Bayesian reasoning: A reply to Lewis and Keren (1999) and Mellers and McGraw (1999). Psychological Review-New York, 1999;106:425-430.

44 Rottenstreich Y, Kivetz R. On decision making without likelihood judgment. Organizational Behavior and Human Decision Processes, 2006;101(1):74-88.

45 Hoffrage U, Gigerenzer G, Krauss S, Martignon L. Representation facilitates reasoning: What natural frequencies are and what they are not. Cognition, 2002; 84(3):343-352.

46 McDaniels TL, Gregory RS. A framework for structuring cross-cultural research in risk and decision making. Journal of Cross-Cultural Psychology, 1991;22(1):103-128.

47 Golec J, Tamarkin M. Bettors love skewness, not risk, at the horse track. Journal of Political Economy, 1998;106(1):205-225.

48 Ebert S. On Skewed Risks in Economic Models and Experiments. Working Paper Available at SSRN 1962783. 2013. Available from: http://ssrn.com/abstract=1962783 or http://dx.doi.org/10.2139/ssrn.1962783

49 Weber EU. Risk attitude and preference. Wiley Interdisciplinary Reviews: Cognitive Science, 2010;1(1):79-88. 
50 Harrison GW, Rutström EE. Expected utility theory and prospect theory: One wedding and a decent funeral. Experimental Economics, 2009;12(2):133-158.

51 Wakker PP. Message to referees who want to embark on yet another discussion of the random-lottery incentive system for individual choice [Internet]. Eurasmus University Rotterdam. 2007 Jan [updated 2008 Feb; cited 2013 Nov 11]. Available from: http://people.few.eur.nl/wakker/miscella/debates/randomlinc.htm.

52 Holt CA, Laury SK. Risk aversion and incentive effects. American Economic Review, 2002;92(5):1644-1655.

53 Lee J. The effect of the background risk in a simple chance improving decision model. Journal of Risk and Uncertainty, 2008;36(1):19-41.

54 Harrison GW. Rutström EE. Risk aversion in the laboratory. Research in Experimental Economics, 2008;12:41-196.

55 Goodwin P, Önkal D, Thomson M. Do forecasts expressed as prediction intervals improve production planning decisions? European Journal of Operational Research, 2010;205(1):195-201.

56 Few S. Information dashboard design. O'Reilly. 2006.

57 Gyrd-Hansen D, Kristiansen IS, Nexøe J, Nielsen JB. How do individuals apply risk information when choosing among health care interventions? Risk Analysis, 2003;23(4):697-704.

58 Racing for Change. Racing for Change reveals "free week" findings. [Press release] 2010 Apr [cited 2010 Apr]. Available from: http://www.racingforchange.co.uk/news/newsitem.aspx?content=61 (2010). 


\section{Table 1}

The three price formats for each of the choices in each race and the associated objective payoffs and likelihoods.

\begin{tabular}{|c|c|c|c|c|c|c|}
\hline \multirow[t]{2}{*}{ Race } & \multirow[t]{2}{*}{ Choice } & \multirow{2}{*}{$\begin{array}{l}\text { Format } \\
\text { Probs. }{ }^{1}\end{array}$} & \multirow{2}{*}{$\begin{array}{l}\text { Format } \\
\text { Odds }\end{array}$} & \multirow{2}{*}{$\begin{array}{l}\text { Format } \\
\text { Returns }\end{array}$} & \multirow[t]{2}{*}{ Variance } & \multirow[t]{2}{*}{ Skewness } \\
\hline & & & & & & \\
\hline \multirow[t]{2}{*}{1} & $F$ & $.555 \dot{5}$ & $4 / 5$ & $\$ 1.80$ & 0.80 & -0.16 \\
\hline & $L$ & $.444 \dot{4}$ & $5 / 4$ & $\$ 2.25$ & 1.25 & 0.31 \\
\hline \multirow[t]{2}{*}{2} & $F$ & .6000 & $4 / 6$ & $\$ 1.67$ & 0.67 & -0.22 \\
\hline & $L$ & .4000 & $6 / 4$ & $\$ 2.50$ & 1.50 & 0.75 \\
\hline \multirow[t]{2}{*}{3} & $F$ & $.666 \dot{6}$ & $1 / 2$ & $\$ 1.50$ & 0.50 & -0.25 \\
\hline & $L$ & $.333 \dot{3}$ & $2 / 1$ & $\$ 3.00$ & 2.00 & 2.00 \\
\hline \multirow[t]{2}{*}{4} & $F$ & .6923 & $4 / 9$ & $\$ 1.44$ & 0.44 & -0.25 \\
\hline & $L$ & .3077 & $9 / 4$ & $\$ 3.25$ & 2.25 & 2.81 \\
\hline \multirow[t]{2}{*}{5} & $F$ & .8000 & $1 / 4$ & $\$ 1.25$ & 0.25 & -0.19 \\
\hline & $L$ & .2000 & $4 / 1$ & $\$ 5.00$ & 4.00 & 12.00 \\
\hline \multirow[t]{2}{*}{6} & $F$ & .9000 & $1 / 9$ & $\$ 1.11$ & 0.11 & -0.10 \\
\hline & $L$ & .1000 & $9 / 1$ & $\$ 10.00$ & 9.00 & 72.00 \\
\hline \multirow[t]{2}{*}{7} & $F$ & .9524 & $1 / 20$ & $\$ 1.05$ & 0.05 & -0.05 \\
\hline & $L$ & .0476 & $20 / 1$ & $\$ 21.00$ & 20.00 & 380.00 \\
\hline
\end{tabular}




\section{Table 2}

Estimates for the random effects parameters (that capture individual differences in subjective expected value and risk attitude) and for the fixed effects (that capture the effect of risk behavior factors) for Model 2: perceived risk-attitude model.

\begin{tabular}{lcccc}
\hline Random Effects & Type & Variance & Standard & \\
(Individual Differences) & & & Deviation & \\
& & & & \\
& Subjective EV $\left(a_{j}\right)$ & 2.408 & 1.55 & \\
& Risk Attitude $\left(b_{j}\right)$ & 11.193 & 3.346 & \\
\hline Participant ${ }_{j}$ & Estimate & Std. E & z value & Sig. \\
\hline Fixed Effects (RBFs) & 0.105 & 0.195 & 0.537 & .591 \\
\hline Intercept) & 0.831 & 0.403 & 2.061 & $.040 *$ \\
FPD & 0.049 & 0.203 & 0.244 & .807 \\
Format(Odds) & 0.247 & 0.213 & 1.161 & .245 \\
LPD $\times$ Format(Odds) & -1.681 & 0.396 & -4.244 & $<.001 * * *$ \\
LPD $\times$ Format(Probabilities) & -4.758 & 0.464 & -10.252 & $<.001 * * *$ \\
\hline
\end{tabular}

${ }^{* * *}$ significant at $.001,{ }^{* *}$ significant at $.01,{ }^{*}$ significant at .05 . 


\section{Table 3}

A comparison of the degree to which Models 1-4 adequately explain the risky choices of participants. Models are ordered by increasing complexity. The degree to which each model accounts for the risky choices of participants is compared with the simpler model above (e.g., Model 2 vs. Model 1). Significant improvements in Log Likelihoods are tested using Chi-squared tests with the difference in the number of parameters between the models as the degrees of freedom.

\begin{tabular}{lcccccc}
\hline Model & Model & $\Delta$ AIC & Log & Chi Sq & Chi Df & Sig. \\
& Df & \multicolumn{7}{c}{ Likelihood } \\
& 5 & -9.13 & -1866.6 & 11.14 & 1 & $<.001^{* * *}$ \\
\hline Model 1 & 9 & -358.20 & -1683.5 & 366.20 & 4 & $<.001^{* * *}$ \\
Model 2 & 15 & -6.02 & -1680.5 & 5.98 & 6 & .425 \\
Model 3 & 21 & -13.5 & -1667.7 & 25.53 & 6 & $<.001^{* * *}$ \\
Model 4 & & & & & & \\
\hline
\end{tabular}

${ }^{* * *}$ significant at $.001,{ }^{* *}$ significant at $.01,{ }^{*}$ significant at .05 . 


\section{Table 4}

ANOVA table (type 3 hypothesis definition from SAS Theory) for Model 4: Dependence Hypothesis Model).

\begin{tabular}{|c|c|c|c|c|}
\hline & Numerator DF & Denominator DF & F value $^{1}$ & Sig. \\
\hline$\overline{\mathrm{LPD}}$ & 1 & 139 & 7.92 & $.006^{* *}$ \\
\hline Format & 2 & 2685 & 2.21 & .110 \\
\hline Culture & 1 & 139 & 1.55 & .214 \\
\hline Experience & 1 & 139 & 0.23 & .631 \\
\hline LPD $\times$ Format & 2 & 2685 & 28.73 & $<.001^{* * *}$ \\
\hline LPD $\times$ Culture & 1 & 139 & 0.83 & .364 \\
\hline Format $\times$ Culture & 2 & 2685 & 6.04 & $.002^{* *}$ \\
\hline LPD×Experience & 1 & 139 & 0.09 & .759 \\
\hline Format $\times$ Experience & 2 & 2685 & 5.44 & $.004^{* *}$ \\
\hline LPD $\times$ Format $\times$ Culture & 2 & 2685 & 4.50 & $.011 *$ \\
\hline LPD $\times$ Format×Experience & 2 & 2685 & 1.90 & .150 \\
\hline
\end{tabular}

${ }^{1}$ The F statistics is calculated based on Satterthwaite's approximation for denominator degrees of freedom.

${ }^{* * *}$ significant at $.001,{ }^{* *}$ significant at $.01,{ }^{*}$ significant at .05 . 


\section{Table 5}

Estimates for the random effects parameters that model individual differences in subjective expected value and risk attitude and the estimates for the fixed effects that model the risk behavior factors (RBFs) for Model 5: Best Fitting Model.

\begin{tabular}{|c|c|c|c|c|}
\hline $\begin{array}{l}\text { Random Effects } \\
\text { (Individual Differences) }\end{array}$ & Type & Variance & $\begin{array}{l}\text { Standard } \\
\text { Deviation }\end{array}$ & \\
\hline \multirow[t]{2}{*}{ Participant $_{j}$} & Subjective EV $\left(a_{j}\right)$ & 2.47 & 1.57 & \\
\hline & Risk Attitude $\left(b_{j}\right)$ & 11.72 & 3.42 & \\
\hline Fixed Effects (RBFs) & Estimate & Std. E & $\mathrm{z}$ value & Sig. \\
\hline (Intercept) & -0.090 & 0.285 & -0.319 & .750 \\
\hline LPD & 0.808 & 0.538 & 1.501 & .133 \\
\hline Format(Odds) & 0.033 & 0.286 & 0.144 & .909 \\
\hline Format(Probabilities) & 1.223 & 0.304 & 3.687 & $<.001 * * *$ \\
\hline Culture(Asian) & 0.025 & 0.399 & 0.063 & .950 \\
\hline Experience(High) & 0.420 & 0.261 & 1.610 & .107 \\
\hline LPD $\times$ Format (Odds) & -1.399 & 0.526 & -2.662 & $.008^{* *}$ \\
\hline LPD $\times$ Format(Probabilities) & -5.747 & 0.634 & -9.065 & $<.001 * * *$ \\
\hline LPD $\times$ Culture(Asian) & 0.104 & 0.828 & 0.126 & .900 \\
\hline Format(Odds) $\times$ Culture(Asian) & -0.021 & 0.411 & -0.050 & .960 \\
\hline Format(Probabilities) $\times$ Culture(Asian) & -1.372 & 0.436 & -3.145 & $.002 * *$ \\
\hline Format(Odds) $\times$ Experience(High) & 0.068 & 0.215 & 0.316 & .752 \\
\hline Format(Probabilities) $\times$ Experience $($ High $)$ & -0.672 & 0.237 & -2.833 & $.005^{* *}$ \\
\hline LPD $\times$ Format $($ Odds $) \times$ Culture $($ Asian $)$ & -0.702 & 0.806 & -0.870 & .384 \\
\hline $\begin{array}{l}\text { LPD } \times \text { Format }(\text { Probabilities }) \times \\
\text { Culture }(\text { Asian })\end{array}$ & 2.185 & 0.941 & 2.322 & $.020 *$ \\
\hline
\end{tabular}


(a) Independence Model - No Interactions

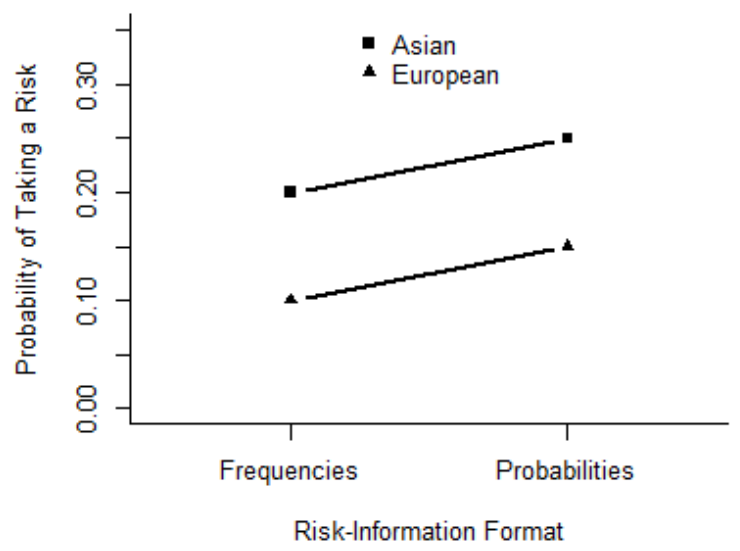

(c) Dependence Model - Group Effect Neurtralized

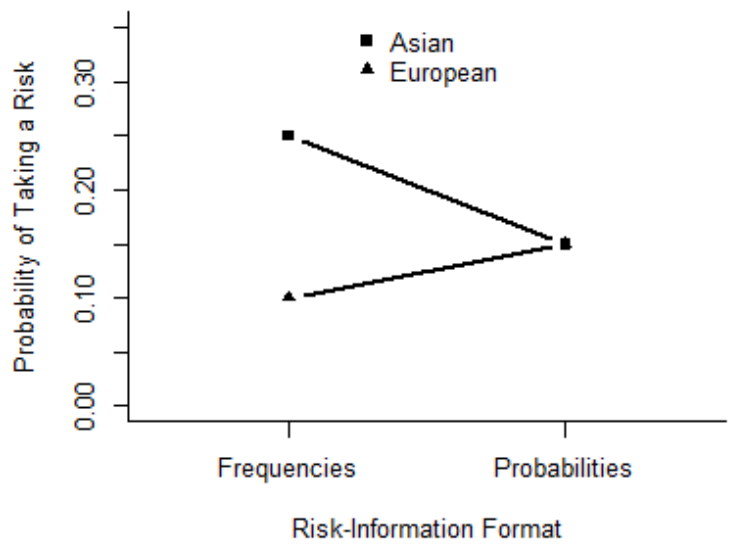

(b) Dependence Model - Group Effect Exaggerated

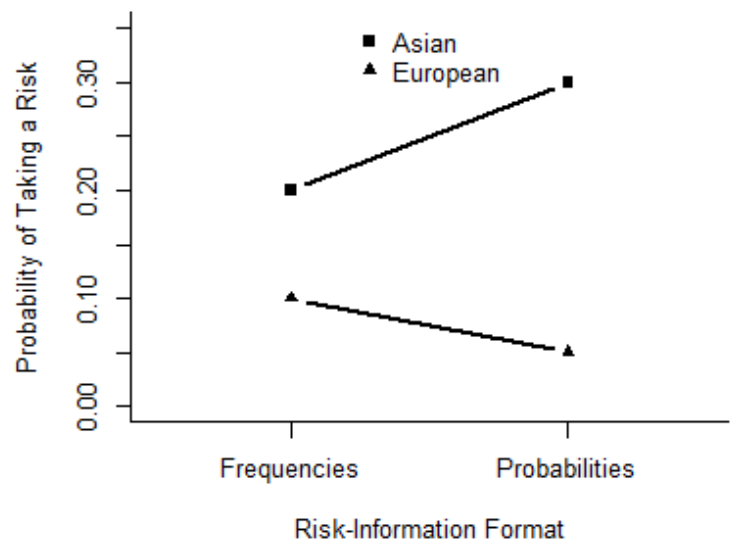

(d) Dependence Model - Group Effect Reversed

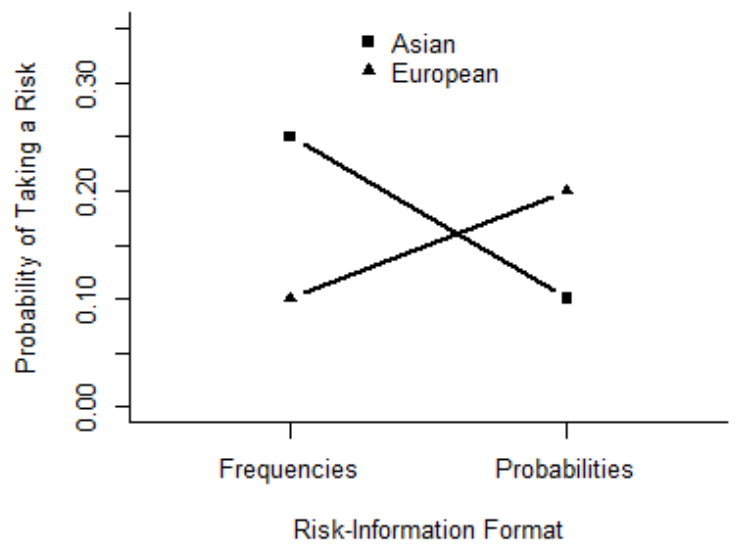

Figure 1. (a) shows a dissociation between the group effects and the framing effects on risktaking propensity; (b-d) show possible interactions between group and framing effects. 

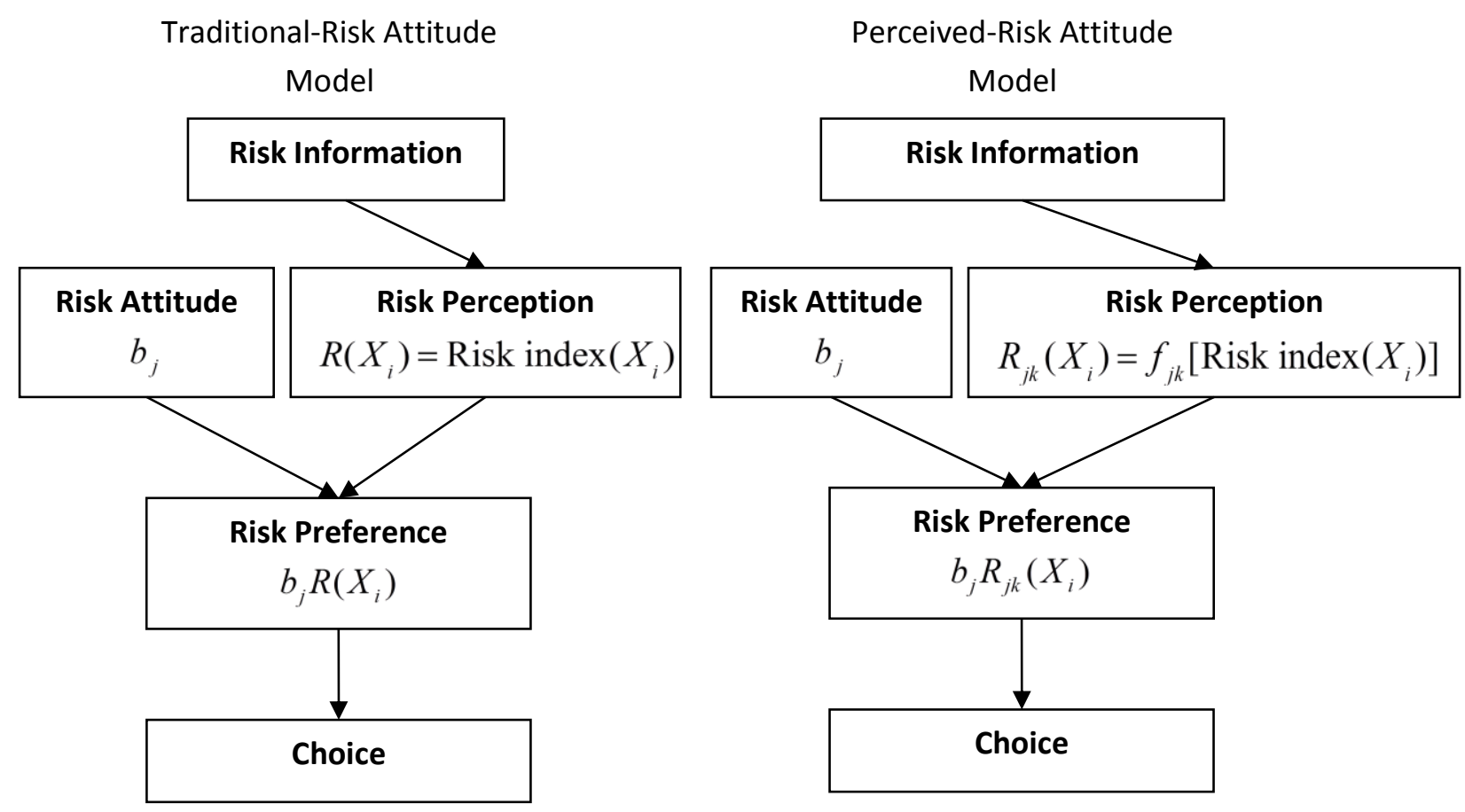

Figure 2. The Traditional-Risk Attitude and Perceived-Risk-Attitude Models of Risk-Preference.

In this figure, $b$ is the risk attitude parameter and $R$ is the perceived magnitude of the risk of choice $X_{i}$ by individual $j$ in the context of $k$ (such as a particular risk-pricing format or for a particular cultural group). 
(a)

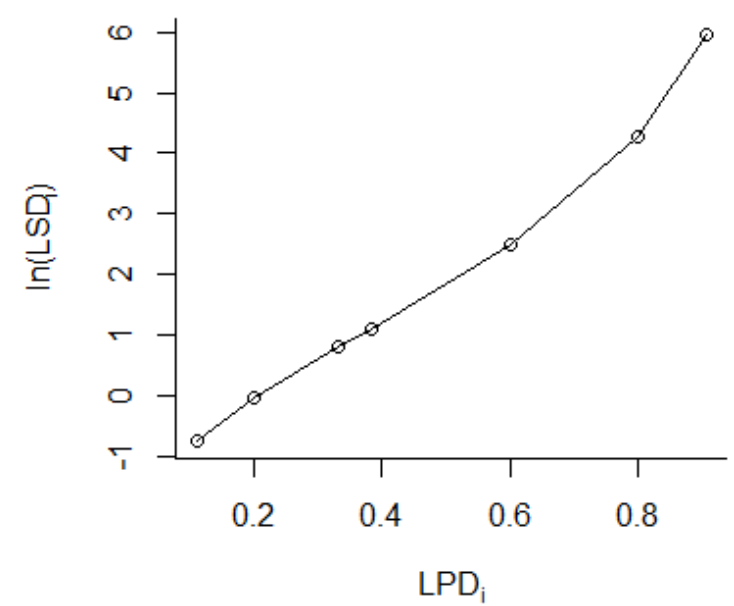

(b)

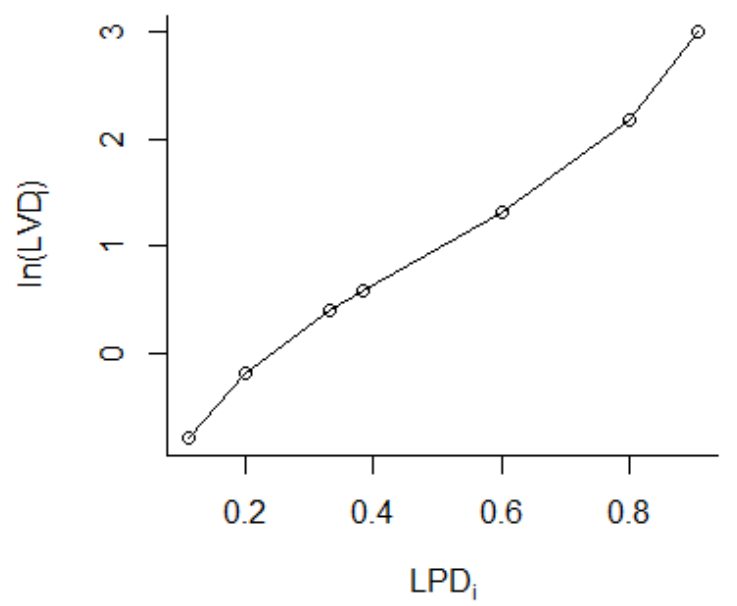

Figure 3. (a) shows the monotonic relationship between the lottery skewness differential $\left(\mathrm{LSD}_{\mathrm{i}}\right.$, shown here as a natural $\log$ transformation, $\ln \left(\operatorname{LSD}_{\mathrm{i}}\right)$, to more clearly display the relationship) and the lottery probability differential $\left(\operatorname{LPD}_{\mathrm{i}}\right)$ for each choice, $i$. (b) shows the monotonic relationship between the lottery variance differential $\left(\operatorname{LVD}_{\mathrm{i}}\right.$, shown here as a natural log transformation, $\left.\ln \left(\operatorname{LVD}_{\mathrm{i}}\right)\right)$ and the lottery probability differential $\left(\mathrm{LPD}_{\mathrm{i}}\right)$ for each choice, $i$. 
(a) Odds

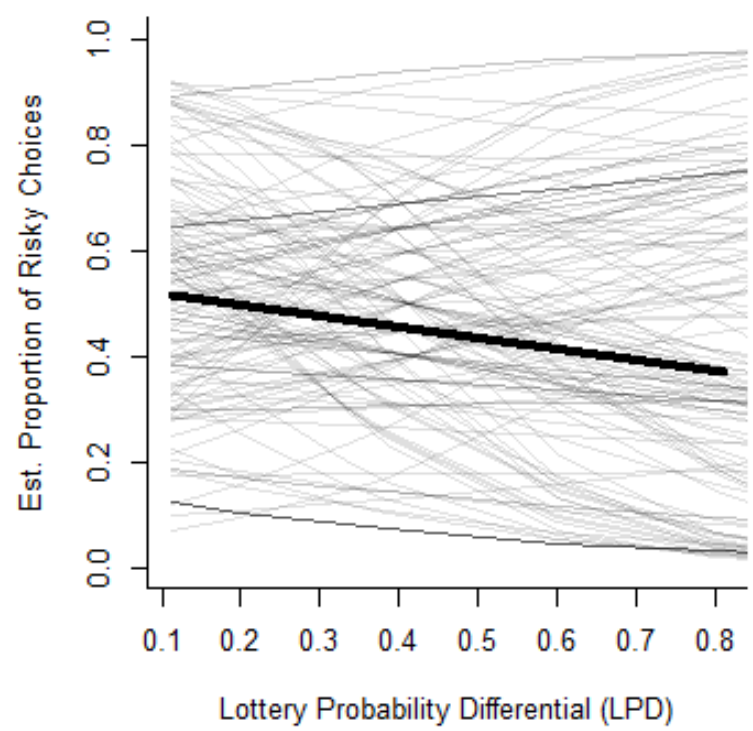

(b) Probabilities

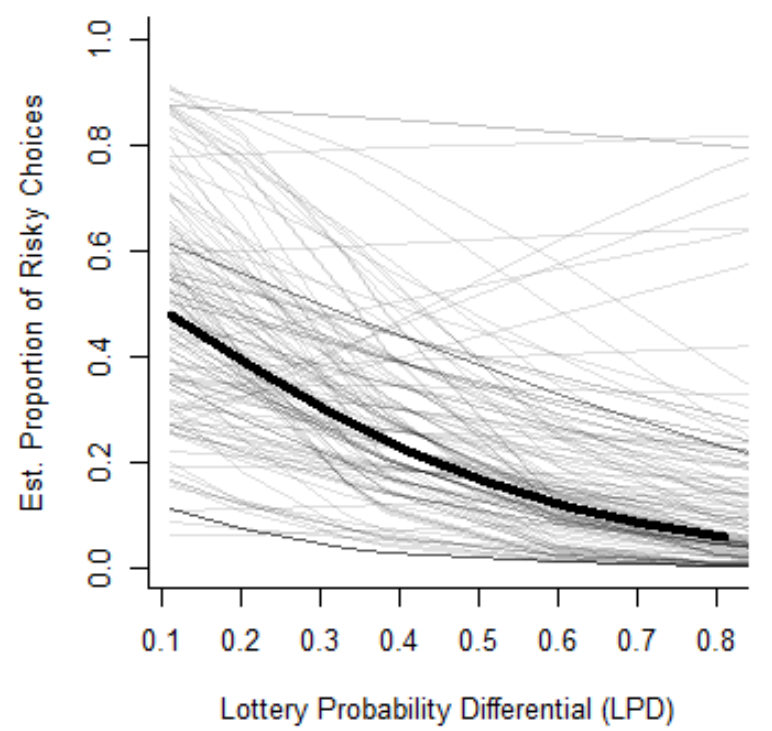

(c) Returns

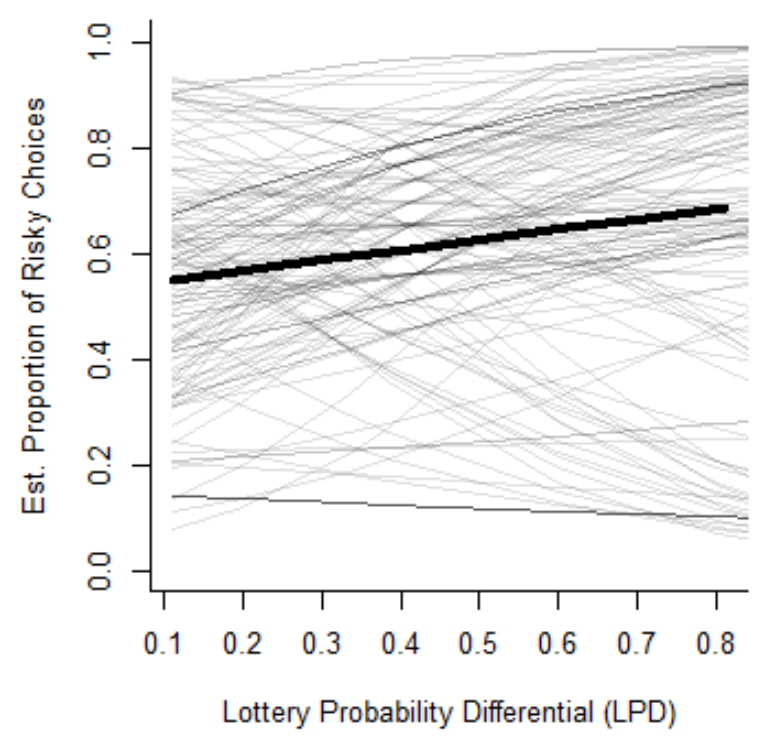

Figure 4. Risky choice behavior of participants modeled according to the perceived risk-attitude model (Model 2).

The figure shows the estimated proportion of risky (vs. safer) choices (based on the perceived risk-attitude model) for each participant (light grey lines) under each risk-pricing format (a-c) as LPD increases. The thick black lines in each graph indicate the fixed factor effects for participants under each risk-pricing format as LPD increases, 
(a) Odds

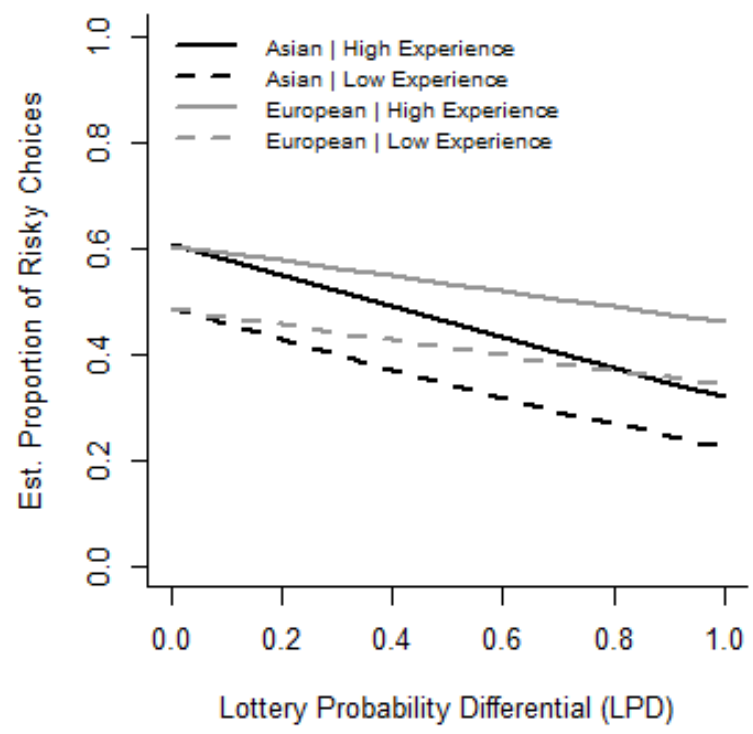

(b) Probabilities

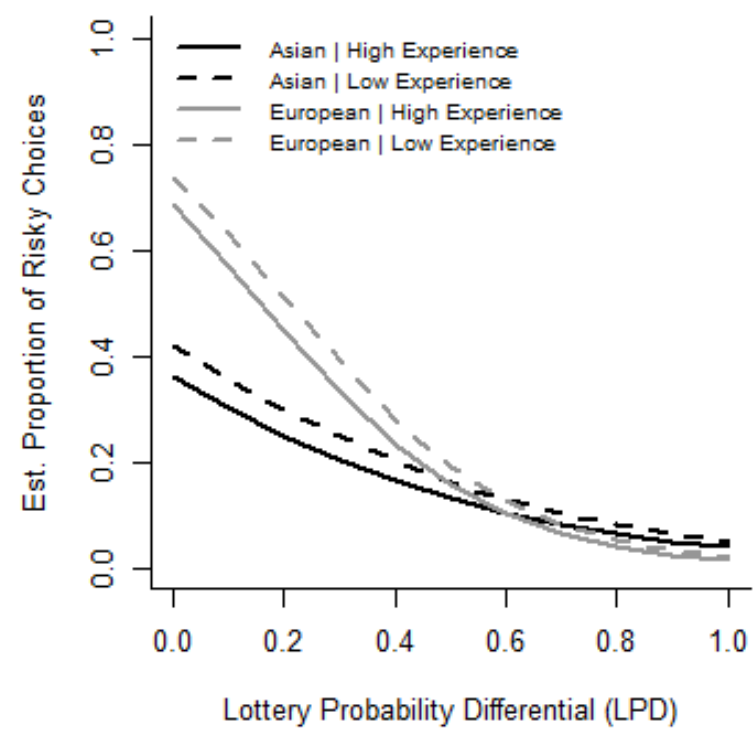

(c) Returns

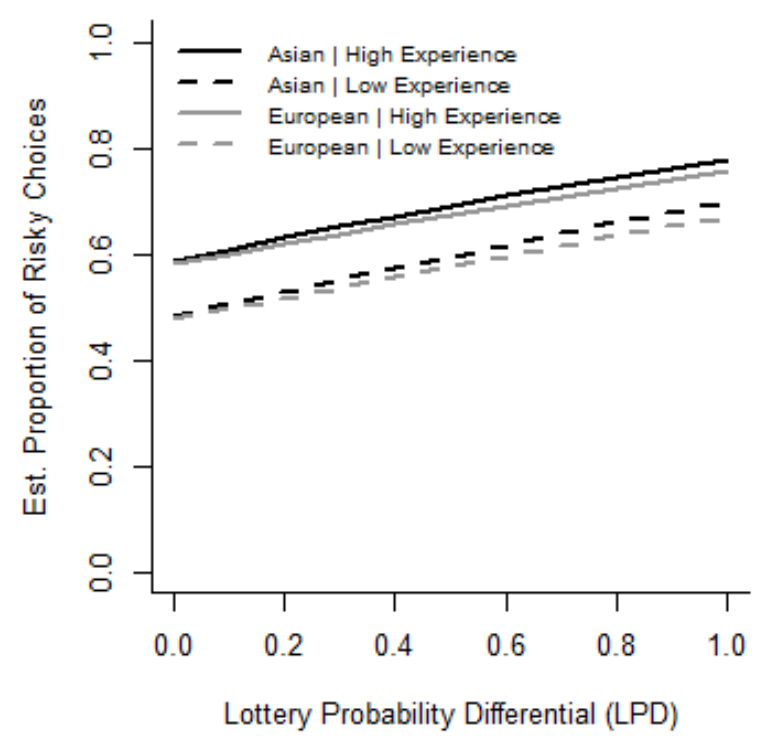

Figure 5. The risky choice behavior of the four cultural background/gambling experience groups (AHE, ALE, EHE and ELE) modeled using the best fit model (Model 5). 


\section{APPENDICES}

\section{Appendix A1}

\section{Introduction}

Placing a bet with a bookmaker means that you are willing to stake a certain fixed amount of money against the bookmaker to predict the outcome of a race correctly. If your horse wins, then the bookmaker will give you back your stake and also an additional sum of money. If you are wrong then you lose your stake. The amount won depends on the likelihood that your horse will win. i.e., in a two horse race, the horse that is unlikely to win (the longshot) will offer a higher payout than the horse than it most likely to win (favorite).

In this experiment you will be given a number of two horse races and you will be asked to decide which horse you would like to bet on. You must decide which horse you would like to place your (hypothetical) $\$ 1$ stake on by marking the box that corresponds to your choice in each race (e.g., see below):

\begin{tabular}{|c|c|c|}
\multicolumn{1}{c}{ Tick } & \multicolumn{1}{c}{ Runners } & $\begin{array}{c}\text { Odds } \\
\text { Offered }\end{array}$ \\
\hline & Horse 1 & $\mathbf{2 0} / \mathbf{1}$ \\
\hline & Horse 2 & $1 / 20$ \\
\hline
\end{tabular}

The information indicating which horse is the favorite and which is the longshot will be either in probabilities, odds, or returns. The meaning of each type of information will be fully explained as you are given the races.

Continue through the booklet reading all the information carefully. 


\title{
Appendix A1 cont.
}

Please read carefully.

\section{Probabilities}

Probabilities indicate the chance that a horse will win. As at least one horse will win the race, the two probabilities in a two horse race will always add up to one.

\author{
For example \\ Horse 1: 0.75 \\ Horse 2: 0.25
}

\section{$\underline{\text { Chance }}$}

Horse 1 has a 0.75 probability, or a $75 \%$ chance that it will finish first. Therefore, it is the favorite. Horse 2 has a 0.25 probability, or a $25 \%$ chance of winning, and as such is the longshot .

\section{Winnings}

Under these probabilities, you would win 3 times and lose once in every four races, if you bet on the favorite. However, the odds on the favorite take this high winning probability into account. This means that if you put $\$ 1$ on the favorite you would win $(1 / 0.75)-1=33$ cents (i.e. you would get back $\$ 1.33$ which includes your $\$ 1$ stake)

If you bet on the longshot you would win 1 and lose 3 in every four races. However, the odds on the longshot take this lower winning probability into account. This means that if you put $\$ 1$ on the longshot you would win $(1 / 0.25)-1=3$ dollars back each time it won (i.e you would get back $\$ 4$ ).

\section{Summary}

In summary, you could potentially win more by betting on the longshot. However, the more money you could win, the less likely the horse will win. The favorite will win more often, yet the returns for an individual bet will be smaller in amount. 


\title{
Appendix A1 cont.
}

\section{Odds}

\section{Please read carefully}

Odds indicate the total you could win if you bet a given stake. Odds tell you how much you could win and also provide an estimate of the chance of the horse winning.

The first number suggests the amount you would win if you bet a stake equal to the second number. Also, the first number tells you how many times the horse is likely to lose and the second number tells you how often it is likely to win in a given number of races (equal to the sum of the first and second number).

\author{
For example: \\ Horse 1:5/2 \\ Horse 2: $2 / 5$
}

\section{Chance}

The larger the first number and the smaller the second, the more unlikely it is that the horse will win. In this case Horse 1 is likely to win 2 times, and lose 5 times, out of 7 races $(5+2)$ Whereas, the smaller the first number and the higher the second number, the more likely it is that the horse will win. In this case, Horse 2 is likely to win 5 times, but lose 2 times, out of 7 races $(2+5)$.

\section{Winnings}

In this case, the bookmaker believes Horse 2 to be the favorite and more likely to win. As a result the bookmaker offers $2 / 5$ on the favorite and $5 / 2$ on the longshot .

This means that if you were to place a $\$ 2$ stake on Horse 1 you would win $\$ 5$ if the horse won (i.e you will be returned $\$ 5$ winnings plus your original stake of $\$ 2$ ). Whereas, the favorite (horse 2) is more likely to win and so you would have to place a $\$ 5$ bet in order to win $\$ 2$ if horse 2 won the race (and your original stake would also be returned).

As you are only allowed to bet $\$ 1$ on each race you would win $\$ 5 / 2=\$ 2.50$ on top of your $\$ 1$ stake (i.e. you would receive back $\$ 3.50$ ) on the longshot if it won, and $\$ 2$ / $5=40$ cents on top of your $\$ 1$ stake (i.e. you would receive back $\$ 1.40$ ) if you bet on the favorite and it won.

\section{Summary}

In summary the first number in the odds tells you how much you win if you bet a stake equal to the second number. The first number also tells you how often it is likely to lose and the second how often it is likely to win (out of a number of races equal to the sum of the first and second number in the odds). 


\section{Appendix A1 cont.}

\section{Returns}

\section{Please read carefully}

Returns indicate exactly how much you would collect from the bookmaker including the $\$ 1$ stake if your selected horse won the race.

For example

Horse 1: 3.50

Horse 2: 1.82

\section{Chance}

In this case, Horse 1 is the longshot as you would win the most if it won. Whereas, the favorite is Horse 2 and you would win the least if it won. The larger the return, the less likely is it to win. The smaller the return, the more likely it is to win. You can determine the probabilities of the horses winning the race by dividing 1 by the return of the longshot, i.e.

Probability of Horse 1 (longshot) winning $=1 / 3.50=0.28$

So, Horse 1 would win $28 \%$ of the time

and Horse 2 would win $72 \%$ of the time ( $1-0.28=.72$ )

\section{$\underline{\text { Winnings }}$}

If you placed 1 dollar on a horse you would be given back the returns figure in dollars. So a $\$ 1$ bet on Horse 1 would return $\$ 3.50$ to you if it won (this figure includes your original $\$ 1$ stake)and a $\$ 1$ bet on Horse 2 would return $\$ 1.82$ to you if it won ((this figure includes your original \$1 stake).

\section{Summary}

The higher the 'returns' number the more you could win but the less likely it is that the horse will win the race. One divided by the return of the longshot indicates its probability of winning and 1 minus the longshot's probability indicates the favorite's probability of winning. The figures displayed tell you exactly the amount in dollars that you would receive if you bet on that horse and it wins. 


\section{Appendix A2}

A binary lottery, $h$, such as those examined in this paper, with payoff structure $(\mathbf{X}, \mathbf{q})$, offers the prospect of a payoff of $x_{1}$ with probability $q_{h}$ and a payoff of $x_{0}$ with probability $1-q_{h}$. The skewness, or third moment about the mean $\left(M_{h}^{3}\right)$, for this lottery is given by $E[\mathbf{X}-E(\mathbf{X})]^{3}$. This is defined as:

$$
p_{h}\left[x_{1}-\left(p_{h} x_{1}-\left(1-p_{h}\right) x_{o}\right)\right]^{3}+\left(1-p_{h}\right)\left[x_{0}-\left(p_{h} x_{1}-\left(1-p_{h}\right) x_{o}\right)\right]^{3} \text {. }
$$

The equivalent-mean risky choices we examine are designed such that $x_{0}=0$ and $E(\mathbf{X})=1$.

Consequently, when the probability of payoff $x_{1}$ is given by $p_{h}$ (and $x_{1}$, is necessarily equal to $1 / p_{h}$ ) then

$$
M_{h}^{3}=p_{h}\left[\frac{1}{p_{h}}-1\right]^{3}+\left(1-p_{h}\right)[-1]^{3}=\left(\frac{1-3 p_{h}+2 p_{h}^{2}}{p_{h}{ }^{2}}\right)=\frac{\left(1-p_{h}\right)\left(1-2 p_{h}^{2}\right)}{p_{h}^{2}} .
$$

This accords with the result given in Golec and Tamarkin (1998), who determine the skewness of the return of a horse, $h$, in a race with objective and subjective probabilities of winning as $p_{h}$ and $b_{h}$, respectively, and $t$ as the track take, as follows:

$$
M_{h}^{3}=\frac{p_{h}\left(1-p_{h}\right)\left(1-2 p_{h}\right)(1-t)^{3}}{b_{h}^{3}}
$$

In our races, we do not include a track take, thus the term $(1 t)^{3}=1$ can be omitted;

$$
M_{h}^{3}=\frac{p_{h}\left(1-p_{h}\right)\left(1-2 p_{h}\right)}{b_{h}^{3}} .
$$

We also assume $p_{h}=b_{h}$ as participants are informed that the odds/returns/probabilities provided for horse $h$ represent its actual probability of winning the race. Consequently, we can simply use $p_{h}$ to represent the likelihood of a given horse winning the race (i.e. $p_{h}$ equals $P_{i}$ and $1-P_{i}$ for alternatives (horses) $C_{i}$ and $C_{i}^{\prime}$, respectively). Thus, for our purposes a skewness function, $S\left(p_{h}\right)$, for horse $h$ is simply a function of the probability of horse $h$ winning the race, as follows: 


$$
S\left(p_{h}\right)=M_{h}^{3}=\frac{\left(1-p_{h}\right)\left(1-2 p_{h}\right)}{p_{h}^{2}}
$$

\section{Appendix A3}

Rounding errors in races 4 and 7 could have resulted in expected values not summing to precisely 1 in these races if calculated on the basis of the probabilities presented. However, expected values calculated using these rounded probabilities (race 4: favorite $=0.9999889$, longshot $=1.000025 ;$ race 7 : favorite $=1.00002$, longshot $=0.9996)$ were not more predictive of participants choices in a mixed model than assuming expected values of 1 for these races (i.e., the intercept), $F(1,2838)=0.83, p=.400$. Therefore, we concluded that participants were not able to discern this edge in these choices as a result of the rounding error.

\section{Appendix A4}

ANOVA table (type 3 hypothesis definition from SAS Theory) for the independence model of gender effects.

\begin{tabular}{lcrrc}
\hline & Numerator DF & Denominator DF & $\mathrm{F}_{\text {value }}$ & Sig. \\
\hline LPD & 1 & 140 & 9.71 & $.002^{* *}$ \\
Format & 2 & 2693 & 0.69 & .499 \\
Gender & 1 & 140 & 0.03 & .864 \\
LPD×Format & 2 & 2693 & 34.33 & $<.001^{* * *}$ \\
LPD×Gender & 1 & 140 & 0.15 & .696 \\
\hline
\end{tabular}

${ }^{* * *}$ significant at $<.001,{ }^{* *}$ significant at $<.01,{ }^{*}$ significant at $<.05$, . significant at $<.1$. 


\section{Appendix A5}

ANOVA table (type 3 hypothesis definition from SAS Theory) for the dependence model of gender effects.

\begin{tabular}{|c|c|c|c|c|}
\hline & Numerator DF & Denominator DF & F value $^{1}$ & Sig. \\
\hline$\overline{L P D}$ & 1 & 140 & 9.71 & $.002^{* *}$ \\
\hline Format & 2 & 2689 & 0.73 & .483 \\
\hline Gender & 1 & 140 & 0.03 & .864 \\
\hline LPD $\times$ Format & 2 & 2689 & 34.37 & $<.001^{* * *}$ \\
\hline LPD $\times$ Gender & 1 & 140 & 0.15 & .696 \\
\hline Format $\times$ Gender & 2 & 2689 & 0.10 & .909 \\
\hline LPD $\times$ Format $\times$ Gender & 2 & 2689 & 0.25 & .782 \\
\hline
\end{tabular}

${ }^{1}$ The F statistics are calculated based on Satterthwaite's approximation for denominator degrees of freedom.

*** significant at .001, ${ }^{* *}$ significant at $.01,{ }^{*}$ significant at .05 . 